Sharif University of Technology
Scientia Iranica
Transactions E: Industrial Engineering
wCIEw.scientiairanica.com

Research Note

\title{
Induced generalized interval neutrosophic Shapley hybrid operators and their application in multi-attribute decision making
}

\author{
P.D. Liu*, G.L. Tang and W.L. Liu \\ School of Management Science and Engineering, Shandong University of Finance and Economics, Jinan Shandong 250014, China.
}

Received 1 December 2015; received in revised form 19 February 2016; accepted 18 July 2016

\author{
KEYWORDS \\ Multi-attribute \\ decision making; \\ Interval neutrosophic \\ set; \\ Aggregation operator; \\ Cross entropy; \\ Generalized Shapley \\ function.
}

\begin{abstract}
With respect to the interval neutrosophic Multi-Attribute Decision-Making (MADM) problems, the MADM method is developed based on some interval neutrosophic aggregation operators. Firstly, the Induced Generalized Interval Neutrosophic Hybrid Arithmetic Averaging (IGINHAA) operator and the Induced Generalized Interval Neutrosophic Hybrid Geometric Mean (IGINHGM) operator are proposed, which can weight all the input arguments and their ordered positions. Further, regarding the situation where the input elements are interdependent, the Induced Generalized Interval Neutrosophic Shapley Hybrid Arithmetic Averaging (IGINSHAA) operator and the Induced Generalized Interval Neutrosophic Shapley Hybrid Geometric Mean (IGINSHGM) operator are proposed, which are extensions of IGINHAA and IGINHGM operators, respectively, and some properties of these given operators are investigated. Furthermore, the interval neutrosophic cross entropy, which is an extension of single-valued neutrosophic cross entropy, is defined, and the models based on the interval neutrosophic cross entropy and generalized Shapley function are respectively constructed to determine the optimal fuzzy measures on the attribute and ordered sets. Finally, an approach to interval neutrosophic MADM with interactive conditions and incomplete known weight information is proposed based on these given operators, and a practical example is shown to verify the practicality and feasibility of the new approach.
\end{abstract}

(C) 2017 Sharif University of Technology. All rights reserved.

\section{Introduction}

Since Neutrosophic Set (NS), which is a generalization of fuzzy set [1], vague set [2], intuitionistic fuzzy set [3], tautological set [4], and so on, was introduced by Smarandache [4], it has been applied in many different areas, such as medical diagnosis $[5,6]$, imaging processing [7], pattern recognition [8], and decisionmaking problems [9-11]. It should be noted that the words "neutrosophy" and "neutrosophic" were invented by Smarandache [4]. Etymologically, "neutroso-

*. Corresponding author. Tel.: +86531 82222188;

E-mail address: Peide.liu@gmail.com (P.D. Liu) phy" (noun) means knowledge of neutral thought while "neutrosophic" (adjective) means having the nature of or the characteristic of Neutrosophy. Because NS consists of three completely independent parts, which are truth-membership degree, indeterminacy-membership, degree and falsity-membership degree, it is very suitable to capture the incomplete, indeterminate, and inconsistent information. Now, NS has attracted more and more attentions [12-17]. Wang et al. [18] defined the Single-Valued Neutrosophic Set (SVNS) as a subclass of the NS. Majumdar and Samanta [19] introduced normalized Hamming distance and normalized Euclidian distance between two SVNSs, and they further presented the similarity between two SVNSs and 
the entropy of a SVNS. Ye [20] defined the single-valued neutrosophic weighted cross entropy and applied it to the MADM problem. Pătraşcu [21] presented two types of neutrosophic entropy as extensions of Kaufman's formula and Kosko's formula, respectively. Biswas et al. [22] developed a modified GRA method for the single-valued neutrosophic MADM problems with completely unknown weight information, and entropy of the SVNS is used to obtain the weight information. Biswas et al. [23] developed an extended TOPSIS method for the neutrosophic MADM problems. In fact, in some complex decision environment, it is insufficient to express the truth-membership degree, indeterminacymembership degree, and falsity membership degree by crisp values. Wang et al. [24] utilized interval numbers to denote these three parts and defined the Interval Neutrosophic Set (INS) as an instance of NS. Chi and Liu [25] developed an extended TOPSIS method to solve MADM problems with interval neutrosophic information, and the maximization deviation method was used to determine the attribute weights.

The aggregation operators are important tools to process the MADM problems [26-30]. With respect to neutrosophic information, Ye [31] proposed the SingleValued Neutrosophic Weighted Geometric Averaging (SVNWGA) operator and applied it to solve singlevalued neutrosophic decision-making problems. Liu and Wang [32] proposed the Single-Valued Neutrosophic Normalized Weighted Bonferroni Mean (SVNNWBM) operator which has the reducibility, idempotency, and commutativity, and utilized it to handle the MADM problems with correlated attributes. Zhang et al. [33] defined the operational and comparison rules of INSs and proposed two interval neutrosophic aggregation operators, including Interval Neutrosophic Number Weighted Averaging Aggregation (INNWAA) operator and Interval Neutrosophic Number Weighted Geometric Aggregation (INNWGA) operator to handle the interval neutrosophic MADM problems. Ye [34] proposed an approach to the MADM problems based on Interval Neutrosophic Number Ordered Weighted Averaging (INNOWA) operator and the Interval Neutrosophic Number Ordered Weighted Geometric (INNOWG) operator. There are some differences between aforementioned interval neutrosophic aggregation operations. INNWAA and INNWGA operators only weight the input arguments themselves, but ignore their ordered positions. INNOWAA and INNOWGA operators only take into account the ordered positions of the interval neutrosophic arguments, but do not consider the arguments themselves. In addition, these above interval neutrosophic number aggregation operators cannot reflect the correlations among the given arguments.

In INNOWAA and INNOWGA operators, we can see that the reordering process depends on the values of the interval neutrosophic arguments, and these two operators could not consider the importance of the aggregated arguments. However, in some real applications, this reordering may not meet our interests. Sometimes, the decision-makers may want to order the arguments based on some other associated variables instead of the aggregated arguments. To overcome these shortcomings, the Induced Generalized Interval Neutrosophic Hybrid Arithmetic Averaging (IGINHAA) operator and the Induced Generalized Interval Neutrosophic Hybrid Geometric Mean (IGINHGM) operator are proposed in this paper. In addition, the aforementioned interval neutrosophic number operators only consider the addition of the importance of individual input arguments. However, in real decision making, the arguments (i.e., attributes) are often not independent. To address these situations, the Induced Generalized Interval Neutrosophic Shapley Hybrid Arithmetic Averaging (IGINSHAA) operator and Induced Generalized Interval Neutrosophic Shapley Hybrid Geometric Mean (IGINSHGM) operator are proposed in this paper. These two operators can not only consider the importance of input arguments and their ordered positions, but also, overall, take into account the correlations among them and their ordered positions. Furthermore, due to time pressure, lack of knowledge or data, and the expert's limited expertise, the weight information in the MADM problems is usually incompletely known or completely unknown. Models based on the cross entropy of the INNs are constructed to determine the attribute weight information. As a very important goal, a method of interval neutrosophic MADM with interactive criteria and incompletely known weight information is developed.

The remainder of this paper is constructed as follows. In Section 2, some basics in regard to INS are introduced, and the cross entropy of INSs is defined. In Section 3, we propose IGINHAA and IGINHGM operators and investigate their several proprieties. In Section 4, we review some basic concepts, such as the fuzzy measure and generalized Shapley function; moreover, we propose IGINSHAA and IGINSHGM operators. In Section 5, the models based on cross entropy of INSs are constructed to obtain the attribute weight information, and a method for the neutrosophic MADM is developed. In Section 6, a practical example is shown to verify the effectiveness of the developed method. In Section 7, the conclusions are summarized.

\section{Preliminaries}

\subsection{Interval Neutrosophic Set (INS)}

Definition 1 [33]. Suppose that $X$ is a universe of discourse, with a generic element in $X$ represented by $x$. An INS $\hat{A}$ in $X$ is expressed by: 


$$
\begin{aligned}
\hat{A}= & \left\{\left\{x, T_{\hat{A}}(x), I_{\hat{A}}(x), F_{\hat{A}}(x)\right\} \mid x \in X\right\}, \\
= & \left\{x,\left\{\left[T_{\hat{A}}^{L}(x), T_{\hat{A}}^{U}(x)\right],\left[I_{\hat{A}}^{L}(x), I_{\hat{A}}^{U}(x)\right],\right.\right. \\
& {\left.\left.\left[F_{\hat{A}}^{L}(x), F_{\hat{A}}^{U}(x)\right]\right\} \mid x \in X\right\}, }
\end{aligned}
$$

where functions $T_{\hat{A}}(x), I_{\hat{A}}(x)$, and $F_{\hat{A}}(x)$ are the truthmembership, indeterminacy-membership, and falsitymembership functions, respectively, and they satisfy $T_{\hat{A}}=\left[T_{\hat{A}}^{L}, T_{\hat{A}}^{U}\right] \subseteq[0,1], I_{\hat{A}}=\left[I_{\hat{A}}^{L}, I_{\hat{A}}^{U}\right] \subseteq[0,1], F_{\hat{A}}=$ $\left[F_{\hat{A}}^{L}, F_{\hat{A}}^{U}\right] \subseteq[0,1]$, and $0 \leq T_{\hat{A}}^{U}+I_{\hat{A}}^{U}+F_{\hat{A}}^{U} \leq 3$.

For convenience, we call $\hat{a}=\{T(\hat{a}), I(\hat{a}), F(\hat{a})\}=$ $\left\{\left[T^{L}(\hat{a}), T^{U}(\hat{a})\right],\left[I^{L}(\hat{a}), I^{U}(\hat{a})\right],\left[F^{L}(\hat{a}), F^{U}(\hat{a})\right] t\right\} \quad$ an Interval Neutrosophic Number (INN). Let:

$$
\hat{a}=\{T(\hat{a}), I(\hat{a}), F(\hat{a})\}
$$

and:

$$
\hat{b}=\{T(\hat{b}), I(\hat{b}), F(\hat{b})\}
$$

be any two INNs; the operational laws of them are shown in Box I [33].

Definition 2 [24]. Suppose $\hat{a}=\{T(\hat{a}), I(\hat{a}), F(\hat{a})\}=$ $\left\{\left[T^{L}(\hat{a}), T^{U}(\hat{a})\right],\left[I^{L}(\hat{a}), I^{U}(\hat{a})\right],\left[F^{L}(\hat{a}), F^{U}(\hat{a})\right]\right\}$ is an INN, the complement of $\hat{a}$ is defined as follows:

$$
\begin{gathered}
\hat{a}^{c}=\left\{T^{c}(\hat{a}), I^{c}(\hat{a}), F^{c}(\hat{a})\right\} \\
=\left\{\left[F^{L}(\hat{a}), F^{U}(\hat{a})\right],\left[1-I^{U}(\hat{a}), 1-I^{L}(\hat{a})\right],\right. \\
\left.\quad\left[T^{L}(\hat{a}), T^{U}(\hat{a})\right]\right\} .
\end{gathered}
$$

Definition 3 [35]. Let $\hat{a}=\{T(\hat{a}), I(\hat{a}), F(\hat{a})\}$ and $\hat{b}=\{T(\hat{b}), I(\hat{b}), F(\hat{b})\}$ be any two INNs, then the Euclidean distance between $\hat{a}$ and $\hat{b}$ is defined as shown in Box II.

Definition 4 [33]. Suppose that:

$$
\hat{a}=\{(T(\hat{a}), I(\hat{a}), F(\hat{a}))\}
$$

is an INN, and then:

$$
\begin{aligned}
S(\hat{a})= & {\left[T^{L}(\hat{a})+1-I^{U}(\hat{a})+1-F^{U}(\hat{a}), T^{U}(\hat{a})\right.} \\
& \left.+1-I^{L}(\hat{a})+1-F^{L}(\hat{a})\right], \\
A(\hat{a})= & {\left[\min \left\{T^{L}(\hat{a})-F^{L}(\hat{a}), T^{U}(\hat{a})-F^{U}(\hat{a})\right\},\right.} \\
& \left.\max \left\{T^{L}(\hat{a})-F^{L}(\hat{a}), T^{U}(\hat{a})-F^{U}(\hat{a})\right\}\right], \\
C(\hat{a})= & {\left[T^{L}(\hat{a}), T^{U}(\hat{a})\right], }
\end{aligned}
$$

$\hat{a} \oplus \hat{b}=\left\{\begin{array}{l}{\left[T^{L}(\hat{a})+T^{L}(\hat{b})-T^{L}(\hat{a}) T^{L}(\hat{b}), T^{U}(\hat{a})+T^{U}(\hat{b})-T^{U}(\hat{a}) T^{U}(\hat{b})\right]} \\ {\left[I^{L}(\hat{a}) I^{L}(\hat{a}), I^{U}(\hat{a}) I^{U}(\hat{b})\right],\left[F^{L}(\hat{a}) F^{L}(\hat{b}), F^{U}(\hat{a}) F^{U}(\hat{b})\right]}\end{array}\right\}$

$\hat{a} \otimes \hat{b}=\left\{\begin{array}{l}{\left[T^{L}(\hat{a}) T^{L}(\hat{b}), T^{U}(\hat{a}) T^{U}(\hat{b})\right],} \\ {\left[I^{L}(\hat{a})+I^{L}(\hat{a})-I^{L}(\hat{a}) I^{L}(\hat{a}), I^{U}(\hat{a})+I^{U}(\hat{b})-I^{U}(\hat{a}) I^{U}(\hat{b})\right],} \\ {\left[F^{L}(\hat{a})+F^{L}(\hat{b})-F^{L}(\hat{a}) F^{L}(\hat{b}), F^{U}(\hat{a})+F^{U}(\hat{b})-F^{U}(\hat{a}) F^{U}(\hat{b})\right]}\end{array}\right\}$

$\lambda \hat{a}=\left\{\begin{array}{l}{\left[1-\left(1-T^{L}(\hat{a})\right)^{\lambda}, 1-\left(1-T^{U}(\hat{a})\right)^{\lambda}\right],} \\ {\left[\left(I^{L}(\hat{a})\right)^{\lambda},\left(I^{U}(\hat{a})\right)^{\lambda}\right],\left[\left(F^{L}(\hat{a})\right)^{\lambda},\left(F^{U}(\hat{a})\right)^{\lambda}\right]}\end{array}\right\} \quad \lambda>0$

$(\hat{a})^{\lambda}=\left\{\begin{array}{l}{\left[\left(T^{L}(\hat{a})\right)^{\lambda},\left(T^{U}(\hat{a})\right)^{\lambda}\right],} \\ {\left[1-\left(1-I^{L}(\hat{a})\right)^{\lambda}, 1-\left(1-I^{U}(\hat{a})\right)^{\lambda}\right],\left[1-\left(1-F^{L}(\hat{a})\right)^{\lambda}, 1-\left(1-F^{U}(\hat{a})\right)^{\lambda}\right]}\end{array}\right\} \quad \lambda>0$ 
$d(\hat{a}, \hat{b})=\sqrt{\frac{1}{6}\left(\left|T^{L}(\hat{a})-T^{L}(\hat{b})\right|^{2}+\left|T^{U}(\hat{a})-T^{U}(\hat{b})\right|^{2}+\left|I^{L}(\hat{a})-I^{L}(\hat{b})\right|^{2}+\left|I^{U}(\hat{a})-I^{U}(\hat{b})\right|^{2}+\left|F^{L}(\hat{a})-F^{L}(\hat{b})\right|^{2}+\left|F^{U}(\hat{a})-F^{U}(\hat{b})\right|^{2}\right)}$.

Box II

where $S(\hat{a}), A(\hat{a})$, and $C(\hat{a})$ represent the score function, accuracy function, and certainty function of INN $\hat{a}$, respectively.

For an INN $\hat{a}$, the bigger the truth-membership $T(\hat{a})$ degree is, the smaller the indeterminacymembership $I(\hat{a})$ degree and the falsity-membership $F(\hat{a})$ degree are, and the greater the INN is. As for the accuracy function, the bigger the difference between truth-membership $T(\hat{a})$ and falsity-membership $F(\hat{a})$ is, the surer the statement is. For the certainty function, the bigger the truth-membership $T(\hat{a})$ is, the greater the corresponding INN is.

Based on the score function, accuracy function and certainty function of INNs, the comparison rules of INNs are shown as follows:

Definition 5 [33]. Let $\hat{a}$ and $\hat{b}$ be any two INNs, the comparison rules are provided as follows:

1. If $S(\hat{a}) \geq S(\hat{b})>\frac{1}{2}$, then $\hat{a} \succ \hat{b}$;

2. If $S(\hat{a}) \geq S(\hat{b})=\frac{1}{2}$, and $A(\hat{a}) \geq A(\hat{b})>\frac{1}{2}$, then $\hat{a} \succ \hat{b}$;

3. If $S(\hat{a}) \geq S(\hat{b})=\frac{1}{2}, A(\hat{a}) \geq A(\hat{b})=\frac{1}{2}$, and $C(\hat{a}) \geq$ $C(\hat{b})>\frac{1}{2}$, then $\hat{a} \succ \hat{b}$;

4. If $S(\hat{a}) \geq S(\hat{b})=\frac{1}{2}, A(\hat{a}) \geq A(\hat{b})=\frac{1}{2}$, and $C(\hat{a}) \geq$ $C(\hat{b})=\frac{1}{2}$, then $\hat{a}=\hat{b}$.

\subsection{The cross entropy of INS}

Before introducing the cross entropy and discrimination information measures between the two INNs, we firstly review the notions of cross entropy and discrimination information measures between two fuzzy sets.

Definition 6 [36]. Let:

$$
A=\left(A\left(x_{1}\right), A\left(x_{2}\right), \cdots, A\left(x_{n}\right)\right),
$$

and:

$$
B=\left(B\left(x_{1}\right), B\left(x_{2}\right), \cdots, B\left(x_{n}\right)\right),
$$

be two fuzzy sets in the universe of discourse, $X=$ $\left\{x_{1}, x_{2}, \cdots, x_{n}\right\}$. The cross entropy between $A$ and $B$ is shown as follows:

$$
\begin{aligned}
& H(A, B)=\sum_{i=1}^{n}\left(A\left(x_{i}\right) \log _{2} \frac{A\left(x_{i}\right)}{\left(A\left(x_{i}\right)+B\left(x_{i}\right)\right) / 2}\right. \\
& \left.\quad+\left(1-A\left(x_{i}\right)\right) \log _{2} \frac{1-A\left(x_{i}\right)}{1-\left(A\left(x_{i}\right)+B\left(x_{i}\right)\right) / 2}\right) .
\end{aligned}
$$

However, the cross entropy $H(A, B)$ is not symmetric in regard to its elements; a symmetric discrimination information measure was introduced by Shang et al. [36] and shown as follows:

$$
I(A, B)=H(A, B)+H(B, A) .
$$

According to the cross entropy and discrimination information measures between two fuzzy sets, the cross entropy and discrimination information measures between two interval neutrosophic sets are defined as follows:

Definition 7. Let $\hat{A}=\left(\hat{A}\left(x_{1}\right), \hat{A}\left(x_{2}\right), \cdots, \hat{A}\left(x_{n}\right)\right)$ and $\hat{B}=\left(\hat{B}\left(x_{1}\right), \hat{B}\left(x_{2}\right), \cdots, \hat{B}\left(x_{n}\right)\right)$ be two interval neutrosophic sets in the universe of discourse $X=$ $\left\{x_{1}, x_{2}, \cdots, x_{n}\right\}$. The cross entropy between $\hat{A}$ and $\hat{B}$ is defined as follows:

$$
\begin{aligned}
E(\hat{A}, \hat{B}) & =\sum_{i=1}^{n}\left[\frac{T_{\hat{A}}^{L}\left(x_{i}\right)+T_{\hat{A}}^{U}\left(x_{i}\right)}{2} \log _{2}\right. \\
& \left.\frac{T_{\hat{A}}^{L}\left(x_{i}\right)+T_{\hat{A}}^{U}\left(x_{i}\right)}{\left(T_{\hat{A}}^{L}\left(x_{i}\right)+T_{\hat{A}}^{U}\left(x_{i}\right)+T_{\hat{B}}^{L}\left(x_{i}\right)+T_{\hat{B}}^{U}\left(x_{i}\right)\right) / 2}\right] \\
& +\sum_{i=1}^{n}\left[\left(1-\frac{T_{\hat{A}}^{L}\left(x_{i}\right)+T_{\hat{A}}^{U}\left(x_{i}\right)}{2}\right) \log _{2}\right. \\
& \left.\frac{1-\left(T_{\hat{A}}^{L}\left(x_{i}\right)+T_{\hat{A}}^{U}\left(x_{i}\right)\right) / 2}{1-\left(T_{\hat{A}}^{L}\left(x_{i}\right)+T_{\hat{A}}^{U}\left(x_{i}\right)+T_{\hat{B}}^{L}\left(x_{i}\right)+T_{\hat{B}}^{U}\left(x_{i}\right)\right) / 4}\right] \\
& +\sum_{i=1}^{n}\left[\frac{I_{\hat{A}}^{L}\left(x_{i}\right)+I_{\hat{A}}^{U}\left(x_{i}\right)}{2} \log _{2}\right. \\
& \left.\frac{I_{\hat{A}}^{L}\left(x_{i}\right)+I_{\hat{A}}^{U}\left(x_{i}\right)}{\left(I_{\hat{A}}^{L}\left(x_{i}\right)+I_{\hat{A}}^{U}\left(x_{i}\right)+I_{\hat{B}}^{L}\left(x_{i}\right)+I_{\hat{B}}^{U}\left(x_{i}\right)\right) / 2}\right]
\end{aligned}
$$




$$
\begin{aligned}
& +\sum_{i=1}^{n}\left[\left(1-\frac{I_{\hat{A}}^{L}\left(x_{i}\right)+I_{\hat{A}}^{U}\left(x_{i}\right)}{2}\right) \log _{2}\right. \\
& \left.\frac{1-\left(I_{\hat{A}}^{L}\left(x_{i}\right)+I_{\hat{A}}^{U}\left(x_{i}\right)\right) / 2}{1-\left(I_{\hat{A}}^{L}\left(x_{i}\right)+I_{\hat{A}}^{U}\left(x_{i}\right)+I_{\hat{B}}^{L}\left(x_{i}\right)+I_{\hat{B}}^{U}\left(x_{i}\right)\right) / 4}\right] \\
& +\sum_{i=1}^{n}\left[\frac{F_{\hat{A}}^{L}\left(x_{i}\right)+F_{\hat{A}}^{U}\left(x_{i}\right)}{2} \log _{2}\right. \\
& \left.\frac{\left(F_{\hat{A}}^{L}\left(x_{i}\right)+F_{\hat{A}}^{U}\left(x_{i}\right)+F_{\hat{B}}^{L}\left(x_{i}\right)+F_{\hat{B}}^{U}\left(x_{i}\right)\right) / 2}{F_{\hat{A}}^{L}\left(x_{i}\right)+F_{\hat{H}}^{U}\left(x_{i}\right)}\right] \\
& +\sum_{i=1}^{n}\left[\left(1-\frac{F_{\hat{A}}^{L}\left(x_{i}\right)+F_{\hat{A}}^{U}\left(x_{i}\right)}{2}\right) \log _{2}\right. \\
& \left.\frac{1-\left(F_{\hat{A}}^{L}\left(x_{i}\right)+F_{\hat{A}}^{U}\left(x_{i}\right)+F_{\hat{B}}^{L}\left(x_{i}\right)+F_{\hat{B}}^{U}\left(x_{i}\right)\right) / 4}{1-\left(F_{\hat{A}}^{L}\left(x_{i}\right)+F_{\hat{A}}^{U}\left(x_{i}\right)\right) / 2}\right]
\end{aligned}
$$

where:

$$
\begin{gathered}
\hat{A}=\left\{\left\{x, T_{\hat{A}}(x), I_{\hat{A}}(x), F_{\hat{A}}(x)\right\} \mid x \in X\right\} \\
=\left\{x,\left\{\left[T_{\hat{A}}^{L}(x), T_{\hat{A}}^{U}(x)\right],\left[I_{\hat{A}}^{L}(x), I_{\hat{A}}^{U}(x)\right],\right.\right. \\
\left.\left.\left[F_{\hat{A}}^{L}(x), F_{\hat{A}}^{U}(x)\right]\right\} \mid x \in X\right\}, \\
\hat{B}=\left\{\left\{x, T_{\hat{B}}(x), I_{\hat{B}}(x), F_{\hat{B}}(x)\right\} \mid x \in X\right\} \\
=\left\{x,\left\{\left[T_{\hat{B}}^{L}(x), T_{\hat{B}}^{U}(x)\right],\left[I_{\hat{B}}^{L}(x), I_{\hat{B}}^{U}(x)\right],\right.\right. \\
\left.\left.\left[F_{\hat{B}}^{L}(x), F_{\hat{B}}^{U}(x)\right]\right\} \mid x \in X\right\} .
\end{gathered}
$$

According to Shannon's inequality [37], we can easily prove that $E(\hat{A}, \hat{B}) \geq 0$ and $E(\hat{A}, \hat{B})=0$ if and only if $T_{\hat{A}}(x)=T_{\hat{B}}(x), I_{\hat{A}}(x)=I_{\hat{B}}(x)$ and $F_{\hat{A}}(x)=F_{\hat{B}}(x)$ for any $x_{i} \in X$. In addition, we can easily prove that $E\left(\hat{A}^{c}, \hat{B}^{c}\right)=E(\hat{A}, \hat{B})$, where $\hat{A}^{c}$ and $\hat{B}^{c}$ are the complements of INSs $\hat{A}$ and $\hat{B}$, respectively.

$E(\hat{A}, \hat{B})$ denotes the degree of discrimination of $\hat{A}$ from $\hat{B}$, which can also be named a discrimination for INSs. Since $E(\hat{A}, \hat{B})$ is not symmetric, a modified symmetric discrimination information measures for INSs is defined as follows:

$$
D(\hat{A}, \hat{B})=E(\hat{A}, \hat{B})+E(\hat{B}, \hat{A}) .
$$

The smaller $D(\hat{A}, \hat{B})$ is, the smaller the difference between $\hat{A}$ and $\hat{B}$ is.

\section{Induced generalized interval neutrosophic hybrid aggregation operators based on the additive measures}

In this section, we will propose IGINHAA and IGINHGM operators, which can weight the interval neutrosophic arguments and their ordered positions by the induced variables.

\subsection{IGHAA operator}

The Induced Generalized Hybrid Averaging (IGHA) operator [38] is a generalization of Induced Ordered Weighted Averaging (IOWA) operator and the Hybrid Weighted Averaging (HWA) operator. The HWA operator weights both input arguments and their ordered positions. However, the HWA operator does not have the boundedness and idempotency. Further, Lin et al. [39] proposed a new Hybrid Weighted Arithmetical Averaging (HWAA) operator with the boundedness and idempotency.

Definition 8 [39]. A HWAA operator of dimension $n$ is a mapping HWAA: $R^{n} \rightarrow R$ that has an associated weight vector $w=\left(w_{1}, w_{2}, \cdots, w_{n}\right)^{T}$ with $w_{j} \in[0,1]$ and $\sum_{j=1}^{n} w_{j}=1$, denoted by:

$$
\operatorname{HWAA}\left(a_{1}, a_{2}, \cdots, a_{n}\right)=\frac{\sum_{j=1}^{n} w_{j} \omega_{(j)} a_{(j)}}{\sum_{j=1}^{n} w_{j} \omega_{(j)}},
$$

where $a_{(j)}$ is the $j$ th largest value of $a_{i}(i=1,2, \cdots, n)$, and $\omega=\left(\omega_{1}, \omega_{2}, \cdots, \omega_{n}\right)^{T}$ is the weight vector of $a_{i}(i=1,2, \cdots, n)$ with $\omega_{j} \in[0,1]$ and $\sum_{j=1}^{n} \omega_{j}=1$.

Moreover, Merigo proposed the Induced Generalized Hybrid Averaging (IGHA) operator [38], which is a generalization of HWA operator by utilizing generalized means [40] and order inducing variables. However, the IGHA operator does not have the properties, such as boundedness and idempotency. Based on HWAA operator, Meng et al. [41] proposed an Induced Generalized Hybrid Arithmetical Averaging (IGHAA) operator which has the boundedness and idempotency.

Definition 9 [41]. Suppose that $u_{i}(i=1,2, \cdots, n)$ is a set of order-inducing variables, and $\gamma$ is a parameter with $\gamma \in(0,+\infty)$. An IGHAA operator of dimension $n$ is a mapping IGHAA: $R^{n} \rightarrow R$ on the set of the second arguments of two tuples $<u_{1}, a_{1}>,<u_{2}, a_{2}>, \cdots,<$ $u_{n}, a_{n}>$, such that:

$$
\begin{aligned}
\operatorname{IGHAA} & \left(<u_{1}, a_{1}>,<u_{2}, a_{2}>, \cdots,<u_{n}, a_{n}>\right) \\
= & \left(\frac{\bigoplus_{j=1}^{n} w_{j} \omega_{(j)}^{\gamma} a_{(j)}^{\gamma}}{\sum_{j=1}^{n} w_{j} \omega_{(j)}^{\gamma}}\right)^{\frac{1}{\gamma}},
\end{aligned}
$$


where $a_{(j)}$ is $a_{i}$ value of IGHAA pair $<u_{i}, a_{i}>$ having the $j$ th largest value of $u_{i}, \omega=\left(\omega_{1}, \omega_{2}, \cdots, \omega_{n}\right)^{T}$ is the weight vector of $a_{i}(i=1,2, \cdots, n)$, such that $\omega_{j} \in$ $[0,1], \sum_{j=1}^{n} \omega_{j}=1$, and $w=\left(w_{1}, \cdots, w_{n}\right)^{T}$ is a weight vector on the ordered set $N=\{1,2, \cdots, n\}$, such that $w_{j} \in[0,1], \sum_{j=1}^{n} w_{j}=1$.

\subsection{IGINHAA operator}

The IGHAA operator fails to aggregate interval neutrosophic information; in this section, we will extend it to INNs and propose IGINHAA operator.

Definition 10. Suppose that $\Omega$ is the set of all INNs, $\hat{a}_{i}=\left\{T\left(\hat{a}_{i}\right), I\left(\hat{a}_{i}\right), F\left(\hat{a}_{i}\right)\right\}(i=1,2, \cdots, n)$ is a collection of INNs, $u_{i}(i=1,2, \cdots, n)$ is a set of order-inducing variables, and $\gamma$ is a parameter with $\gamma \in(0,+\infty)$. An IGINHAA operator of dimension $n$ is a mapping IGINHAA: $\Omega^{n} \rightarrow \Omega$ on the set of the second components of 2-tuple arguments $\left\langle u_{1}, \hat{a}_{1}\right\rangle,<$ $u_{2}, \hat{a}_{2}>, \cdots<u_{n}, \hat{a}_{n}>$, such that:

$$
\begin{aligned}
\operatorname{IGINHAA}( & \left.<u_{1}, \hat{a}_{1}>,<u_{2}, \hat{a}_{2}>, \cdots,<u_{n}, \hat{a}_{n}>\right) \\
= & \left(\frac{\bigoplus_{j=1}^{n} w_{j} \omega_{(j)}^{\gamma} \hat{a}_{(j)}^{\gamma}}{\sum_{j=1}^{n} w_{j} \omega_{(j)}^{\gamma}}\right)^{\frac{1}{\gamma}},
\end{aligned}
$$

where $\hat{a}_{(j)}$ is $\hat{a}_{i}$ value of IGINHAA pair $<u_{i}, \hat{a}_{i}>$ having the $j$ th largest value of $u_{i}, \omega=\left(\omega_{1}, \omega_{2}, \cdots, \omega_{n}\right)^{T}$ is the weight vector of $\hat{a}_{i}(i=1,2, \cdots, n)$, such that $\omega_{j} \in[0,1]$ and $\sum_{j=1}^{n} \omega_{j}=1$, and $w=\left(w_{1}, \cdots, w_{n}\right)^{T}$ is a weight vector on the ordered set $N=\{1, \cdots, n\}$, such that $w_{j} \in[0,1], \sum_{j=1}^{n} w_{j}=1$.

However, if there is a tie between $<u_{i}, \hat{a}_{i}>$ and $\left\langle u_{j}, \hat{a}_{j}>\right.$ regarding order-inducing variables such that $u_{i}=u_{j}$, then in this case, we replace the second components of 2-tuple arguments $<u_{i}, \hat{a}_{i}>$ and $<u_{j}, \hat{a}_{j}>$ by their generalized mean $\left(\left(\hat{a}_{i}^{\gamma} \oplus \hat{a}_{j}^{\gamma}\right) / 2\right)^{1 / \gamma}$. If $k$ order-inducing variables are equal, we replace these by $k$ replicas of their generalized mean.

Theorem 1. With the operations of INNs, IGINHAA operator (Eq. (13)) can be transformed into the following form.

$$
\begin{aligned}
& \operatorname{IGINHAA}\left(<u_{1}, \hat{a}_{1}>,<u_{2}, \hat{a}_{2}>, \cdots,<u_{n}, \hat{a}_{n}>\right) \\
& =\left\{\left[\left(1-\prod_{j=1}^{n}\left(1-\left(T^{L}\left(\hat{a}_{(j)}\right)\right)^{\gamma}\right)^{\left.\frac{w_{j} \omega_{(j)}^{\gamma}}{\sum_{j=1}^{n} w_{j} \omega_{(j)}^{\gamma}}\right)^{\frac{1}{\gamma}},}\right.\right.\right.
\end{aligned}
$$

$$
\left.\left(1-\prod_{j=1}^{n}\left(1-\left(T^{U}\left(\hat{a}_{(j)}\right)\right)^{\gamma}\right)^{\frac{w_{j} \omega_{(j)}^{\gamma}}{\sum_{j=1}^{n} w_{j} \omega_{(j)}^{\gamma}}}\right)^{\frac{1}{\gamma}}\right]
$$$$
\left[1-\left(1-\prod_{j=1}^{n}\left(1-\left(1-I^{L}\left(\hat{a}_{(j)}\right)\right)^{\gamma}\right)^{\frac{w_{j} \omega_{(j)}^{\gamma}}{\sum_{j=1}^{n} w_{j} \omega_{(j)}^{\gamma}}}\right)^{\frac{1}{\gamma}}\right.
$$$$
\left.1-\left(1-\prod_{j=1}^{n}\left(1-\left(1-I^{U}\left(\hat{a}_{(j)}\right)\right)^{\gamma}\right)^{\frac{w_{j} \omega_{(j)}^{\gamma}}{\sum_{j=1}^{n} w_{j}^{\gamma} \omega_{(j)}^{\gamma}}}\right)^{\frac{1}{\gamma}}\right],
$$$$
\left[1-\left(1-\prod_{j=1}^{n}\left(1-\left(1-F^{L}\left(\hat{a}_{(j)}\right)\right)^{\gamma}\right){ }_{j=1}^{\frac{w_{j} \omega_{(j)}^{\gamma}}{\sum_{j=1}^{n} w_{j} \omega_{(j)}^{\gamma}}}\right)^{\frac{1}{\gamma}},\right.
$$$$
\left.\left.1-\left(1-\prod_{j=1}^{n}\left(1-\left(1-F^{U}\left(\hat{a}_{(j)}\right)\right)^{\gamma}\right)^{\frac{w_{j} \omega_{(j)}^{\gamma}}{\sum_{j=1}^{n} w_{j} \omega_{(j)}^{\gamma}}}\right)^{\frac{1}{\gamma}}\right]\right\}
$$

whose aggregated value is also an INN.

Proof. Firstly, as shown in Box III, we prove that Eq. (14) holds.

Next, we prove that Eq. (14) is an INN. It is easy to prove the following inequalities:

$$
\begin{aligned}
& {\left[\left(1-\prod_{i=1}^{n}\left(1-\left(T_{\left(\hat{a}_{(j)}\right)}^{L}\right)^{\gamma}\right)^{\frac{{ }_{w_{j} \omega_{(j)}^{\gamma}}}{\sum_{j=1}^{n} w_{j} \omega_{(j)}^{\gamma}}}\right)^{\frac{1}{\gamma}}\right.} \\
& \left.\left(1-\prod_{i=1}^{n}\left(1-\left(T_{\left(\hat{a}_{(j)}\right)}^{U}\right)^{\gamma}\right)^{\frac{w_{j=1} \omega_{(j)}^{\gamma}}{\sum_{j=1}^{n} w_{j}^{\gamma} \omega_{(j)}^{\gamma}}}\right)^{\frac{1}{\gamma}}\right] \subseteq[0,1], \\
& {\left[1-\left(1-\prod_{i=1}^{n}\left(1-\left(1-I_{\left(\hat{a}_{(j)}\right)}^{L}\right)^{\gamma}\right)^{\frac{{ }_{w_{j} \omega_{(j)}^{\gamma}}}{\sum_{j=1}^{n} w_{j} \omega_{(j)}^{\gamma}}}\right)^{\frac{1}{\gamma}}\right.}
\end{aligned}
$$

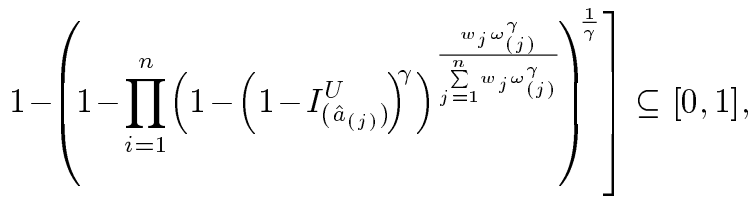




$$
\begin{aligned}
& \operatorname{IGINHAA}\left(<\mu_{1}, \hat{a}_{1}>,<\mu_{2}, \hat{a}_{2}>, \cdots,<\mu_{n}, \hat{a}_{n}>\right) \\
& \left.=\left(\frac{\bigoplus_{j=1}^{n} w_{j} \omega_{(j)}^{\gamma}\left\{\begin{array}{c}
{\left[\left(T_{\left(\hat{a}_{(j)}\right)}^{L}\right)^{\gamma},\left(T_{\left(\hat{a}_{(j)}\right)}^{U}\right)^{\gamma}\right],\left[1-\left(1-I_{\left(\hat{a}_{(j)}\right)}^{L}\right)^{\gamma}, 1-\left(1-I_{\left(\hat{a}_{(j)}\right)}^{U}\right)^{\gamma}\right],} \\
{\left[1-\left(1-F_{\left(\hat{a}_{(j)}\right)}^{L}\right)^{\gamma}, 1-\left(1-F_{\left(\hat{a}_{(j)}\right)}^{U}\right)^{\gamma}\right]}
\end{array}\right.}{\sum_{j=1}^{n} w_{j} \omega_{(j)}^{\gamma}}\right\}\right)^{\frac{1}{\gamma}}
\end{aligned}
$$

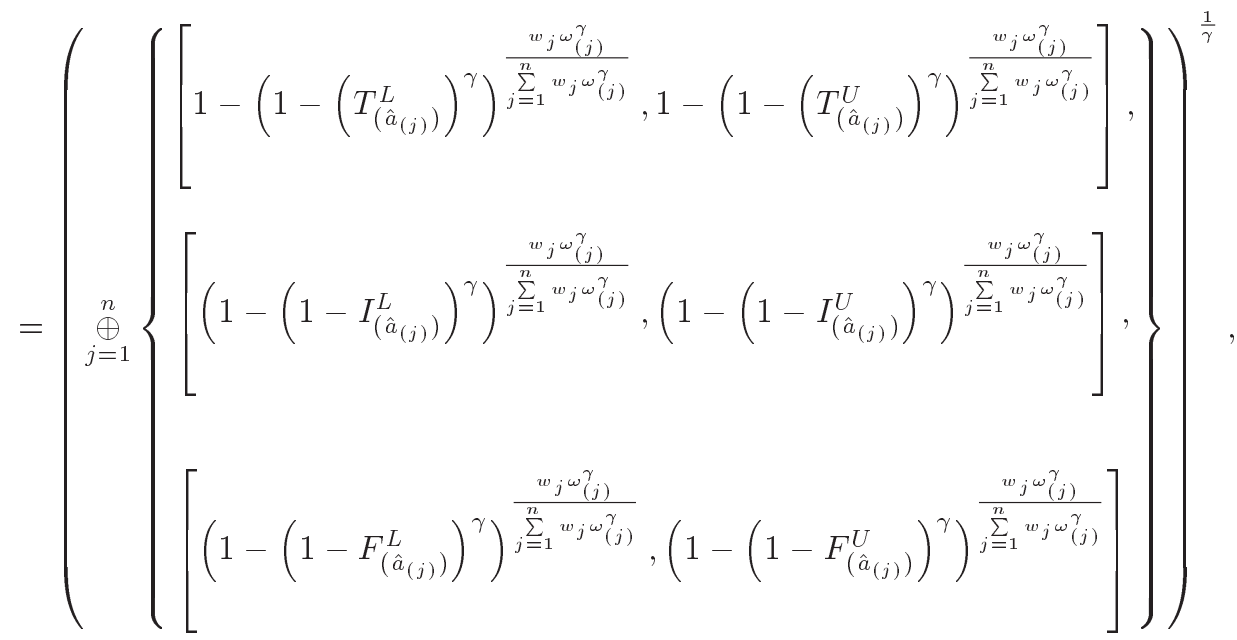

$\operatorname{IGINHAA}\left(<u_{1}, \hat{a}_{1}>,<u_{2}, \hat{a}_{2}>, \cdots,<u_{n}, \hat{a}_{n}>\right)$

$$
\begin{aligned}
& =\left\{\left[\left(1-\prod_{j=1}^{n}\left(1-\left(T^{L}\left(\hat{a}_{(j)}\right)\right)^{\gamma}\right)^{\frac{{ }_{w_{j} \omega_{(j)}^{\gamma}} \sum_{j=1}^{n} w_{j} \omega_{(j)}^{\gamma}}{\gamma}}\right)^{\frac{1}{\gamma}},\left(1-\prod_{j=1}^{n}\left(1-\left(T^{U}\left(\hat{a}_{(j)}\right)\right)^{\gamma}\right)^{\frac{{ }_{w_{j} \omega_{(j)}^{\gamma}} \sum_{j=1}^{n} w_{j} \omega_{(j)}^{\gamma}}{\gamma}}\right)^{\frac{1}{\gamma}}\right]\right.
\end{aligned}
$$

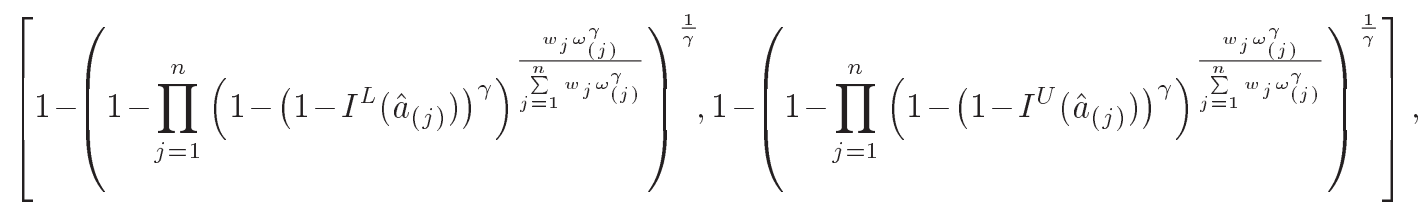

$$
\begin{aligned}
& {\left[1-\left(1-\prod_{j=1}^{n}\left(1-\left(1-F^{L}\left(\hat{a}_{(j)}\right)\right)^{\gamma}\right)\right)_{j=1}^{\frac{w_{j} \omega_{(j)}^{\gamma}}{\sum_{j=1}^{n} w_{j} \omega_{(j)}^{\gamma}}}\right)^{\frac{1}{\gamma}}} \\
& \left.\left.1-\left(1-\prod_{j=1}^{n}\left(1-\left(1-F^{U}\left(\hat{a}_{(j)}\right)\right)^{\gamma}\right)^{\frac{w_{j=1} w_{j} \omega_{(j)}^{\gamma}}{\sum_{j}^{n} w_{j}^{\gamma}(j)}}\right)^{\frac{1}{\gamma}}\right]\right\}
\end{aligned}
$$




$$
\begin{gathered}
{\left[1-\left(1-\prod_{i=1}^{n}\left(1-\left(1-F_{\left(\hat{a}_{(j)}\right)}^{L}\right)^{\gamma}\right)^{\frac{w_{j} \omega_{(j)}^{\gamma}}{\sum_{j=1}^{n} w_{j} \omega_{(j)}^{\gamma}}}\right)^{\frac{1}{\gamma}},\right.} \\
\left.1-\left(1-\prod_{i=1}^{n}\left(1-\left(1-F_{\left(\hat{a}_{(j)}\right)}^{U}\right)^{\gamma}\right)^{\frac{{ }_{w_{j} \omega_{(j)}^{\gamma}}}{\sum_{j=1}^{n} w_{j} \omega_{(j)}^{\gamma}}}\right)^{\frac{1}{\gamma}}\right] \subseteq[0,1] .
\end{gathered}
$$

Thus, it is an INN.

Remark 1. If $\gamma=1$, then IGINHAA operator reduces to the Induced Interval Neutrosophic Hybrid Arithmetic Averaging (IINHAA) operator:

$$
\begin{aligned}
\operatorname{IINHAA}( & \left.<u_{1}, \hat{a}_{1}>,<u_{2}, \hat{a}_{2}>, \cdots,<u_{n}, \hat{a}_{n}>\right) \\
= & \frac{\bigoplus_{j=1}^{n} w_{j} \omega_{(j)} \hat{a}_{(j)}}{\sum_{j=1}^{n} w_{j} \omega_{(j)}} .
\end{aligned}
$$

Furthermore, If $w_{j}=\frac{1}{n}(j=1,2, \cdots, n)$, then we get the Interval Neutrosophic Number Weighted Averaging Aggregation (INNWAA) operator [33].

Remark 2. If $\gamma=2$, then IGINHAA operator reduces to the Induced Interval Neutrosophic Hybrid Quadratic Averaging (IINHQA) operator:

$$
\begin{aligned}
\operatorname{IINHQA}( & \left.<u_{1}, \hat{a}_{1}>,<u_{2}, \hat{a}_{2}>, \cdots,<u_{n}, \hat{a}_{n}>\right) \\
= & \left(\frac{\bigoplus_{j=1}^{n} w_{j} \omega_{(j)}^{2} \hat{a}_{(j)}^{2}}{\sum_{j=1}^{n} w_{j} \omega_{(j)}^{2}}\right)^{\frac{1}{2}} .
\end{aligned}
$$

From these remarks, we can know that IGINHAA operator is the generalized form of IINHAA, INNWAA, and IINHQA operators.

Based on the operational laws of INNs, we shall prove that IGINHAA operator has the following properties.

Theorem 2 (idempotency). Suppose that $\hat{a}_{i}=$ $a=\{T(a), I(a), F(a)\}(i=1,2, \cdots, n)$, then we have:

$\operatorname{IGINHAA}\left(<u_{1}, \hat{a}_{1}>,<u_{2}, \hat{a}_{2}>, \cdots,<u_{n}, \hat{a}_{n}>\right)=\hat{a}$.

Theorem 3 (Commutativity). Suppose that ( $\hat{a}_{1}^{\prime}$, $\left.\hat{a}_{2}^{\prime}, \cdots, \hat{a}_{n}^{\prime}\right)$ is any permutation of $\left(\hat{a}_{1}, \hat{a}_{2}, \cdots, \hat{a}_{n}\right)$, then we get:

$$
\begin{aligned}
& \operatorname{IGINHNAA}\left(<u_{1}, \hat{a}_{1}>,<u_{2}, \hat{a}_{2}>, \cdots,<u_{n}, \hat{a}_{n}>\right) \\
& =\operatorname{IGINHNAA}\left(<u_{1}^{\prime}, \hat{a}_{1}^{\prime}>,<\dot{u}_{2}^{\prime}, \hat{a}_{2}^{\prime}>, \cdots,<u_{n}^{\prime}, \hat{a}_{n}^{\prime}>\right) .
\end{aligned}
$$

Theorem 4 (monotonicity). Suppose that $A=$ $\left(\hat{a}_{1}, \hat{a}_{2}, \cdots, \hat{a}_{n}\right)$ and $\tilde{B}=\left(\hat{b}_{1}, \hat{b}_{2}, \cdots, \hat{b}_{n}\right)$ are two collections of INNs, $\hat{a}_{i}=\left\{T\left(\hat{a}_{i}\right), I\left(\hat{a}_{i}\right), F\left(\hat{a}_{i}\right)\right\}(i=$ $1,2, \cdots, n), \hat{b}_{i}=\left\{T\left(\hat{b}_{i}\right), I\left(\hat{b}_{i}\right), F\left(\hat{b}_{i}\right)\right\}(i=1,2, \cdots, n)$, and $(\cdot)$ is a permutation on $\tilde{A}$ and $\tilde{B}$, respectively, such that:

$$
\begin{array}{ll}
T^{L}\left(\hat{a}_{(i)}\right) \leq T^{L}\left(\hat{b}_{(i)}\right), & T^{U}\left(\hat{a}_{(i)}\right) \leq T^{U}\left(\hat{b}_{(i)}\right), \\
I^{L}\left(\hat{a}_{(i)}\right) \geq I^{L}\left(\hat{b}_{(i)}\right), & I^{U}\left(\hat{a}_{(i)}\right) \geq I^{U}\left(\hat{b}_{(i)}\right), \\
F^{L}\left(\hat{a}_{(i)}\right) \geq F^{L}\left(\hat{b}_{(i)}\right), & F^{U}\left(\hat{a}_{(i)}\right) \geq F^{U}\left(\hat{b}_{(i)}\right),
\end{array}
$$

then we have:

$$
\begin{aligned}
& \operatorname{IGINHAA}\left(<u_{1}, \hat{a}_{1}>,<u_{1}, \hat{a}_{2}>, \cdots,<u_{1}, \hat{a}_{n}>\right) \\
& \leq \operatorname{IGINHAA}\left(<u_{1}, \hat{b}_{1}>,<u_{1}, \hat{b}_{2}>, \cdots,<u_{1}, \hat{b}_{n}>\right) .
\end{aligned}
$$

Theorem 5 (boundedness). Suppose that $\hat{a}_{i}=$ $\left\{T\left(\hat{a}_{i}\right), I\left(\hat{a}_{i}\right), F\left(\hat{a}_{i}\right)\right\}(i=1,2, \cdots, n)$ is a collection of INNs, and:

$$
\begin{aligned}
\hat{a}^{-}=\{[ & \left.\max \left(T^{L}\left(\hat{a}_{i}\right)\right), \max \left(T^{U}\left(\hat{a}_{i}\right)\right)\right], \\
& {\left[\min \left(I^{L}\left(\hat{a}_{i}\right)\right), \min \left(I^{U}\left(\hat{a}_{i}\right)\right)\right], } \\
& {\left.\left[\min \left(F^{L}\left(\hat{a}_{i}\right)\right), \min \left(F^{U}\left(\hat{a}_{i}\right)\right)\right]\right\}, } \\
\hat{a}^{+}=\{[ & {\left[\min \left(T^{L}\left(\hat{a}_{i}\right)\right), \min \left(T^{U}\left(\hat{a}_{i}\right)\right)\right], } \\
& {\left[\max \left(I^{L}\left(\hat{a}_{i}\right)\right), \max \left(I^{U}\left(\hat{a}_{i}\right)\right)\right], } \\
& {\left.\left[\max \left(F^{L}\left(\hat{a}_{i}\right)\right), \max \left(F^{U}\left(\hat{a}_{i}\right)\right)\right]\right\}, }
\end{aligned}
$$

then:

$$
\begin{aligned}
\hat{a}^{-} & \leq \operatorname{IGINHAA}\left(<u_{1}, \hat{a}_{1}>,<u_{2}, \hat{a}_{2}>, \cdots,<u_{n}, \hat{a}_{n}>\right) \\
& \leq \hat{a}^{+} .
\end{aligned}
$$

Limited to the space, the proofs of the properties of IGINHAA operator are omitted here.

\subsection{IGINHGM operator}

In terms of IGINHAA operator and geometric mean, we define an IGINHGM operator.

Definition 11. Suppose that $\Omega$ is the set of all INNs, $\hat{a}_{i}=\left\{T\left(\hat{a}_{i}\right), I\left(\hat{a}_{i}\right), F\left(\hat{a}_{i}\right)\right\} ;(i=1,2, \cdots, n)$ is a collection of INNs, $u_{i}(i=1,2, \cdots, n)$ is a set of order-inducing variables, and $\gamma$ is a parameter with $\gamma \in(0,+\infty)$. An IGINHGM operator of dimension $n$ is a mapping IGINHGM: $\Omega^{n} \rightarrow \Omega$ on the set of the second components of 2-tuple arguments $<u_{1}, \hat{a}_{1}>,<$ 
$u_{2}, \hat{a}_{2}>, \cdots<u_{n}, \hat{a}_{n}>$, such that:

$$
\begin{aligned}
\operatorname{IGINHGM}( & \left.<u_{1}, \hat{a}_{1}>,<u_{2}, \hat{a}_{2}>, \cdots,<u_{n}, \hat{a}_{n}>\right) \\
& =\frac{1}{\gamma}\left(\bigotimes_{i=1}^{n}\left(\gamma \hat{a}_{(j)}\right)^{\frac{w_{j} \omega_{(j)}}{\sum^{n} w_{j} w_{j} \omega}}\right),
\end{aligned}
$$

where $\hat{a}_{(j)}$ is $\hat{a}_{i}$ value of IGINHGM pair $<u_{i}, \hat{a}_{i}>$ having the $j$ th largest value of $u_{i}, \omega=\left(\omega_{1}, \omega_{2}, \cdots, \omega_{n}\right)^{T}$ is the weight vector of $\hat{a}_{i}(i=1,2, \cdots, n)$, such that $\omega_{j} \in[0,1]$ and $\sum_{j=1}^{n} \omega_{j}=1 ; w=\left(w_{1}, w_{2}, \cdots, w_{n}\right)^{T}$ is a weight vector on the ordered set $N=\{1,2, \cdots, n\}$, such that $w_{j} \in[0,1]$ and $\sum_{j=1}^{n} w_{j}=1$.

However, if there is a tie between $\left\langle u_{i}, \hat{a}_{i}>\right.$ and $<u_{j}, \hat{a}_{j}>$ regarding order-inducing variables such that $u_{i}=u_{j}$, then in this case, we replace the second components of 2-tuple arguments $\left\langle u_{i}, \hat{a}_{i}\right\rangle$ and $<u_{j}, \hat{a}_{j}>$ by their generalized geometric mean $\frac{1}{\gamma}\left(\left(\gamma \hat{a}_{i}\right)^{\frac{1}{2}} \otimes\left(\gamma \hat{a}_{i}\right)^{\frac{1}{2}}\right)$. If $k$ items are tied, we replace these by $k$ replicas of their generalized geometric mean.

Theorem 6. With the operations of INNs, IGINHGM operator (Eq. (17)) can be transformed into the following form:

$$
\begin{aligned}
& \operatorname{IGINHGM}\left(<u_{1}, \hat{a}_{1}>,<u_{2}, \hat{a}_{2}>, \cdots,<u_{n}, \hat{a}_{n}>\right) \\
& =\left\{\left[1-\left(1-\prod_{j=1}^{n}\left(1-\left(1-T^{L}\left(\hat{a}_{(j)}\right)\right)^{\gamma}\right)^{\frac{{ }_{j=1} w_{j} \omega_{(j)}}{\sum_{n=1}^{n} w_{j} \omega_{(j)}}}\right)^{\frac{1}{\gamma}},\right.\right. \\
& \left.1-\left(1-\prod_{j=1}^{n}\left(1-\left(1-T^{U}\left(\hat{a}_{(j)}\right)\right)^{\gamma}\right)^{\frac{w_{j} \omega_{(j)}}{\sum_{j=1}^{n} w_{j} \omega_{(j)}}}\right)^{\frac{1}{\gamma}}\right], \\
& {\left[\left(1-\prod_{j=1}^{n}\left(1-\left(I^{L}\left(\hat{a}_{(j)}\right)\right)^{\gamma}\right)^{\frac{w_{j=1}^{n} \omega_{(j)}}{\sum_{j=1} w_{j} \omega_{(j)}}}\right)^{\frac{1}{\gamma}}\right.} \\
& \left.\left(1-\prod_{j=1}^{n}\left(1-\left(I^{U}\left(\hat{a}_{(j)}\right)\right)^{\gamma}\right)^{\frac{w_{j=1}^{w_{j} \omega_{(j)}}}{\sum_{j}^{n} w_{j} \omega}(j)}\right)^{\frac{1}{\gamma}}\right] \\
& {\left[\left(1-\prod_{j=1}^{n}\left(1-\left(F^{L}\left(\hat{a}_{(j)}\right)\right)^{\gamma}\right)^{\frac{w_{j=1}^{n} w_{j} w_{j} \omega_{(j)}}{\sum_{n}^{n}}}\right)^{\frac{1}{\gamma}}\right.} \\
& \left.\left.\left(1-\prod_{j=1}^{n}\left(1-\left(F^{U}\left(\hat{a}_{(j)}\right)\right)^{\gamma}\right)^{\frac{\sum_{j=1}^{w_{j} \omega_{(j)}}}{\sum_{n=1}^{n} w_{j} \omega_{(j)}}}\right)^{\frac{1}{\gamma}}\right]\right\}
\end{aligned}
$$

whose aggregated value is also an INN.
Similar to the proof of Theorem 1, it is easy to get the result.

Remark 3. If $\gamma=1$, then IGINHGM operator reduces to the Induced Interval Neutrosophic Hybrid Geometric Mean (IINHGM) operator:

$$
\begin{aligned}
\operatorname{IINHGM}( & \left.<u_{1}, \hat{a}_{1}>,<u_{2}, \hat{a}_{2}>, \cdots,<u_{n}, \hat{a}_{n}>\right) \\
& =\bigotimes_{i=1}^{n}\left(\hat{a}_{(j)}\right)^{\frac{w_{j=1}^{w_{j} \omega_{(j)}}}{\sum_{j=1}^{n} w_{j} \omega(j)}} .
\end{aligned}
$$

Furthermore, if $w_{j}=\frac{1}{n}(j=1,2, \cdots, n)$, then we get the Interval Neutrosophic Number Weighted Geometric Aggregation (INNWGA) operator [33].

Remark 4. If $\gamma=2$, then IGINHGM operator reduces to the Induced Interval Neutrosophic Hybrid Quadratic Geometric (IINHQG) operator.

$$
\begin{aligned}
\operatorname{IINHQG}( & \left.<u_{1}, \hat{a}_{1}>,<u_{2}, \hat{a}_{2}>, \cdots,<u_{n}, \hat{a}_{n}>\right) \\
& =\frac{1}{2}\left(\bigotimes_{i=1}^{n}\left(2 \cdot \hat{a}_{(j)}\right)^{\frac{\sum_{j=1}^{w_{j} \omega}(j)}{\sum^{n} w_{j} \omega(j)}}\right) .
\end{aligned}
$$

From these remarks, we can know that IGINHGM operator is the generalized form of IINHGM, INNWGA, and IINHQG operators.

Similar to the proofs of the properties of IGINHAA operator, we can prove that IGINHGM operator also has idempotency, commutativity, monotonicity, and boundedness.

\section{The induced generalized interval neutrosophic hybrid aggregation operators based on the Shapley function}

Although IGINHAA and IGINHGM operators can weigh the interval neutrosophic arguments and their ordered positions, they are under the implicit assumption that the aggregated interval neutrosophic arguments are independent. However, in some situations, especially in some decision-making cases, some correlations may exist between the input elements; these two operators cannot process this condition. To address this situation, we shall propose two new aggregation interval neutrosohphic operators, named IGINSHAA and IGINSHGM operators, which not only weight the interval neutrosophic arguments and their ordered positions, but also consider the interactions among them and among their ordered positions.

\subsection{Fuzzy measures and the generalized Shapley function}

Definition 12 [42]. A fuzzy measure on a finite set $N=\{1,2, \cdots, n\}$ is a set function $\mu: P(N) \rightarrow[0,1]$. It satisfies the following conditions: 
1. Boundary: $\mu(\phi)=0, \mu(N)=1$;

2. Monotonicity: If $F, G \in P(N)$ and $F \subseteq G$, then $\mu(F) \leq \mu(G)$, where $P(N)$ is the power set of $N$.

In MADM problems, $\mu(F)$ can be regarded as the importance of attribute set $F$.

The generalized Shapley function was provided by Shapley [43], which is shown as follows:

$$
\varphi_{i}(\mu, N)=\sum_{F \subseteq N \backslash i} \frac{(n-f-1) ! f !}{n !}(\mu(i \cup f)-\mu(f))
$$

$\forall i \in N$

where $\mu$ is a fuzzy measure on $N=\{1,2, \cdots, n\}, n$ and $f$ represent the cardinalities of $N$ and $F$, respectively. From the generalized Shapley function, we know that it is an expectation value of the overall interaction between element $i \in N$ and every combination in $N \backslash i$. From Eq. (21) and Definition 12, it is easy to know that $\left\{\varphi_{i}(\mu, N)\right\}_{i \in N}$ is a weight vector, because $\varphi_{i}(\mu, N) \geq$ 0 for any element $i \in N$ and $\sum_{i=1}^{n} \varphi_{i}(\mu, N)=1$. It should be noted that if there are no interactions between elements, then the Shapley values are equal to their own importance.

\subsection{IGINSHAA operator}

Definition 13. Suppose that $\Omega$ is the set of all INNs, $\hat{a}_{i}=\left\{T\left(\hat{a}_{i}\right), I\left(\hat{a}_{i}\right), F\left(\hat{a}_{i}\right)\right\}(i=1,2, \cdots, n)$ is a collection of INNs, $u_{i}(i=1,2, \cdots, n)$ is a set of order-inducing variables, and $\gamma$ is a parameter with $\gamma \in(0,+\infty)$. An IGINSHAA operator of dimension $n$ is a mapping IGINSHAA: $\Omega^{n} \rightarrow \Omega$ on the set of the second components of 2-tuple arguments $\left.<u_{1}, \hat{a}_{1}\right\rangle,<$ $u_{2}, \hat{a}_{2}>, \cdots<u_{n}, \hat{a}_{n}>$, such that:

$$
\begin{aligned}
\operatorname{IGINSHAA}( & \left.<\mu_{1}, \hat{a}_{1}>,<\mu_{2}, \hat{a}_{2}>, \cdots,<\mu_{n}, \hat{a}_{n}>\right) \\
= & \left(\frac{\bigoplus_{j=1}^{n} \varphi_{j}(\mu, N) \varphi_{(j)}^{\gamma}(\nu, A) \hat{a}_{(j)}^{\gamma}}{\sum_{j=1}^{n} \varphi_{j}(\mu, N) \varphi_{(j)}^{\gamma}(\nu, A)}\right)^{\frac{1}{\gamma}},(2)
\end{aligned}
$$

where $\hat{a}_{(j)}$ is $\hat{a}_{i}$ value of IGINSHAA pair $<u_{i}, \hat{a}_{i}>$ having the $j$ th largest value of $u_{i}, \varphi_{j}(\mu, N)$ is the Shapley value in regard to the associated fuzzy measure $\mu$ on $N=\{1,2, \cdots, n\}$ for $j$ th ordered positions, and $\varphi_{(j)}(\nu, A)$ is the Shapley value in regard to fuzzy measure $\nu$ on $A=\left\{\hat{a}_{1}, \hat{a}_{2}, \cdots, \hat{a}_{n}\right\}$ for $\hat{a}_{(j)}(j=1,2, \cdots, n)$.

However, if there is a tie between $<u_{i}, \hat{a}_{i}>$ and $<$ $u_{j}, \hat{a}_{j}>$ with respect to order-inducing variables such that $u_{i}=u_{j}$, then in this case, we replace the second components of 2-tuple arguments $<u_{i}, \hat{a}_{i}>$ and $<$ $u_{j}, \hat{a}_{j}>$ by their generalized mean $\left(\left(\hat{a}_{i}^{\gamma} \oplus \hat{a}_{j}^{\gamma}\right) / 2\right)^{1 / \gamma}$. If $k$ items are tied, we replace these by $k$ replicas of their generalized mean.

Theorem 7. With the operations of INNs, IGINSHAA operator (Eq. (22)) can be transformed into the form shown in Box IV, whose aggregated valued is also an INN.

Similar to the proof of Theorem 1, it is easy to get the result.

Remark 5. If fuzzy measures $\mu$ and $\nu$ are both additive, then IGINSHAA operator degenerates to IGINHAA operator.

$$
\begin{aligned}
& \operatorname{IGINSHAA}\left(<u_{1}, \hat{a}_{1}>,<u_{2}, \hat{a}_{2}>, \cdots,<u_{n}, \hat{a}_{n}>\right) \\
& =\left\{\left[\left(1-\prod_{j=1}^{n}\left(1-\left(T^{L}\left(\hat{a}_{(j)}\right)\right)^{\gamma}\right)^{\frac{\varphi_{j}(\mu, N) \varphi_{(j)}^{\gamma}(\mu, A)}{\sum_{j=1}^{n} \varphi_{j}(\mu, N) \varphi_{(j)}^{\gamma}(\mu, A)}}\right)^{\frac{1}{\gamma}},\left(1-\prod_{j=1}^{n}\left(1-\left(T^{U}\left(\hat{a}_{(j)}\right)\right)^{\gamma}\right)^{\frac{\varphi_{j}(\mu, N) \varphi_{(j)}^{\gamma}(\mu, A)}{\sum_{j=1}^{n} \varphi_{j}(\mu, N) \varphi_{(j)}^{\gamma}(\mu, A)}}\right)^{\frac{1}{\gamma}}\right]\right. \\
& {\left[1-\left(1-\prod_{j=1}^{n}\left(1-\left(1-I^{L}\left(\hat{a}_{(j)}\right)\right)^{\gamma}\right)_{j=1}^{\frac{\varphi_{j}(\mu, N) \varphi_{(j)}^{\gamma}(\mu, A)}{\sum_{j=1}^{n} \varphi_{j}(\mu, N) \varphi_{(j)}^{\gamma}(\mu, A)}}\right)^{\frac{1}{\gamma}}, 1-\left(1-\prod_{j=1}^{n}\left(1-\left(1-I^{U}\left(\hat{a}_{(j)}\right)\right)^{\gamma}\right)^{\frac{\varphi_{j}(\mu, N) \varphi_{(j)}^{\gamma}(\mu, A)}{\sum_{j=1}^{n} \varphi_{j}(\mu, N) \varphi_{(j)}^{\gamma}(\mu, A)}}\right)^{\frac{1}{\gamma}}\right]} \\
& \left.\left[1-\left(1-\prod_{j=1}^{n}\left(1-\left(1-F^{L}\left(\hat{a}_{(j)}\right)\right)^{\gamma}\right)^{\frac{\varphi_{j}(\mu, N) \varphi_{(j)}^{\gamma}(\mu, A)}{\sum_{j=1}^{n} \varphi_{j}(\mu, N) \varphi_{(j)}^{\gamma}(\mu, A)}}\right)^{\frac{1}{\gamma}}, 1-\left(1-\prod_{j=1}^{n}\left(1-\left(1-F^{U}\left(\hat{a}_{(j)}\right)\right)^{\gamma}\right)^{\frac{\varphi_{j}(\mu, N) \varphi_{(j)}^{\gamma}(\mu, A)}{\sum_{j=1}^{n} \varphi_{j}(\mu, N) \varphi_{(j)}^{\gamma}(\mu, A)}}\right)^{\frac{1}{\gamma}}\right]\right\}
\end{aligned}
$$


Remark 6. If $\gamma=1$, then IGINSHAA operator reduces to the Induced Interval Neutrosophic Shapley Hybrid Arithmetic Averaging (IINSHAA) operator:

$$
\begin{aligned}
\operatorname{IINSHAA}( & \left.<\mu_{1}, \hat{a}_{1}>,<\mu_{2}, \hat{a}_{2}>, \cdots,<\mu_{n}, \hat{a}_{n}>\right) \\
= & \frac{\bigoplus_{j=1}^{n} \varphi_{j}(\mu, N) \varphi_{(j)}(\nu, A) \hat{a}_{(j)}}{\sum_{j=1}^{n} \varphi_{j}(\mu, N) \varphi_{(j)}(\nu, A)}
\end{aligned}
$$

Remark 7. If $\gamma=2$, then IGINSHAA operator reduces to the Induced Interval Neutrosophic Shapley Hybrid Quadratic Averaging (IINSHQA) operator:

$$
\begin{aligned}
\operatorname{IGINSHAA}( & \left.<\mu_{1}, \hat{a}_{1}>,<\mu_{2}, \hat{a}_{2}>, \cdots,<\mu_{n}, \hat{a}_{n}>\right) \\
= & \left(\frac{\bigoplus_{j=1}^{n} \varphi_{j}(\mu, N) \varphi_{(j)}^{2}(\nu, A) \hat{a}_{(j)}^{2}}{\sum_{j=1}^{n} \varphi_{j}(\mu, N) \varphi_{(j)}^{2}(\nu, A)}\right)^{\frac{1}{2}} \cdot(25)
\end{aligned}
$$

Similar to the proofs of the properties of IGINHAA operator, we can see that IGINSHAA operator has properties, such as idempotency, commutativity, monotonicity, and boundedness.

\subsection{IGINSHGM operator}

Definition 14. Suppose that $\Omega$ is the set of all INNs, $\hat{a}_{i}=\left\{T\left(\hat{a}_{i}\right), I\left(\hat{a}_{i}\right), F\left(\hat{a}_{i}\right)\right\}(i=1,2, \cdots, n)$ is a collection of INNs, $u_{i}(i=1,2, \cdots, n)$ is a set of order-inducing variables, and $\gamma$ is a parameter with $\gamma \in(0,+\infty)$. An IGINSHGM operator of dimension $n$ is a mapping IGINSHGM: $\Omega^{n} \rightarrow \Omega$ on the set of the second components of 2-tuple arguments $\left.<u_{1}, \hat{a}_{1}\right\rangle,<$ $u_{2}, \hat{a}_{2}>, \cdots<u_{n}, \hat{a}_{n}>$, such that:

$$
\operatorname{IGINSHGM}\left(<u_{1}, \hat{a}_{1}>,<u_{2}, \hat{a}_{2}>, \cdots,<u_{n}, \hat{a}_{n}>\right)
$$

$$
=\frac{1}{\gamma}\left(\bigotimes_{i=1}^{n}\left(\gamma \hat{a}_{(j)}\right)^{\frac{\varphi_{j}(\mu, N) \varphi_{(j)}(\nu, A)}{\sum_{j=1}^{n} \varphi_{j}(\mu, N) \varphi_{(j)}(\nu, A)}}\right),
$$

where $\hat{a}_{(j)}$ is $\hat{a}_{i}$ value of IGINSHGM pair $<u_{i}, \hat{a}_{i}>$ having the $j$ th largest value of $u_{i}, \varphi_{j}(\mu, N)$ is the Shapley value in regard to the associated fuzzy measure $\mu$ on $N=\{1,2, \cdots, n\}$ for $j$ th ordered positions, and $\varphi_{(j)}(\nu, A)$ is the Shapley value in regard to fuzzy measure $\nu$ on $A=\left\{\hat{a}_{1}, \hat{a}_{2}, \cdots, \hat{a}_{n}\right\}$ for $\hat{a}_{(j)}(j=1,2, \cdots, n)$.

However, if there is a tie between $\left\langle u_{i}, \hat{a}_{i}\right\rangle$ and $<u_{j}, \hat{a}_{j}>$ with respect to order-inducing variables such that $u_{i}=u_{j}$, then in this case, we replace the second components of 2-tuple arguments $\left\langle u_{i}, \hat{a}_{i}\right\rangle$ and $\left\langle u_{j}, \hat{a}_{j}\right\rangle$ by their generalized geometric mean $\frac{1}{\gamma}\left(\left(\gamma \hat{a}_{i}\right)^{\frac{1}{2}} \otimes\left(\gamma \hat{a}_{i}\right)^{\frac{1}{2}}\right)$. If $k$ items are tied, we replace these by $k$ replicas of their generalized geometric mean.

Theorem 8. With the operations of INNs, IGINSHGM operator (Eq. (26)) can be transformed into the form shown in Box V, whose aggregated value is also an INN.

Similar to the proof of Theorem 1, it is easy to get the result.

Remark 8. If $\mu$ and $\nu$ are both additive, then IGINSHGM operator degenerates to IGINHGM operator.

Remark 9. If $\gamma=1$, then IGINSHGM operator reduces to the Induced Interval Neutrosophic Shapley

$\operatorname{IGINSHGM}\left(<\mu_{1}, \hat{a}_{1}>,<\mu_{2}, \hat{a}_{2}>, \cdots,<\mu_{n}, \hat{a}_{n}>\right)$

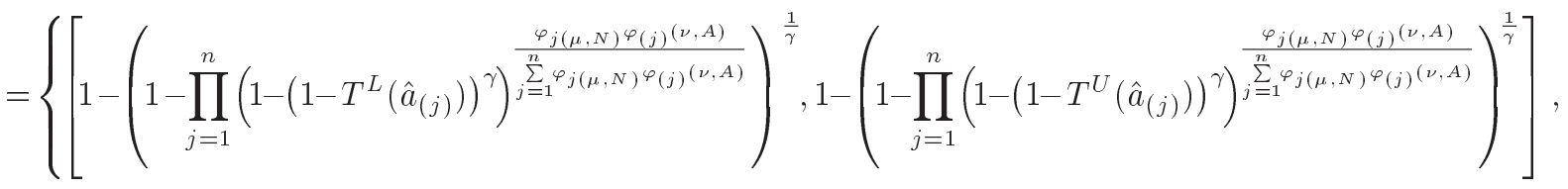

$$
\begin{aligned}
& {\left[\left(1-\prod_{j=1}^{n}\left(1-\left(I^{L}\left(\hat{a}_{(j)}\right)\right)^{\gamma}\right)^{\frac{\varphi_{j}(\mu, N) \varphi_{(j)}(\nu, A)}{\sum_{j=1}^{n} \varphi_{j(\mu, N)^{\varphi}(j)}(\nu, A)}}\right)^{\frac{1}{\gamma}},\left(1-\prod_{j=1}^{n}\left(1-\left(I^{U}\left(\hat{a}_{(j)}\right)\right)^{\gamma}\right)^{\frac{\varphi_{j}(\mu, N)^{\varphi} \varphi_{(j)}(\nu, A)}{\sum_{j=1}^{n} \varphi_{j(\mu, N)^{\varphi}(j)}(\nu, A)}}\right)^{\frac{1}{\gamma}}\right],} \\
& \left.\left[\left(1-\prod_{j=1}^{n}\left(1-\left(F^{L}\left(\hat{a}_{(j)}\right)\right)^{\gamma}\right)^{\frac{\varphi_{j(\mu, N)} \varphi_{(j)}(\nu, A)}{\sum_{j=1}^{n} \varphi_{j(\mu, N)} \varphi_{(j)}(\nu, A)}}\right)^{\frac{1}{\gamma}},\left(1-\prod_{j=1}^{n}\left(1-\left(F^{U}\left(\hat{a}_{(j)}\right)\right)^{\gamma}\right)^{\frac{\varphi_{j(\mu, N)} \varphi_{(j)}(\nu, A)}{\sum_{j=1}^{n} \varphi_{j(\mu, N)} \varphi_{(j)}(\nu, A)}}\right)^{\frac{1}{\gamma}}\right]\right\}
\end{aligned}
$$


Hybrid Geometric Mean (IINSHGM) operator:

$$
\begin{aligned}
\operatorname{IGINSHGM}( & \left.<u_{1}, \hat{a}_{1}>,<u_{2}, \hat{a}_{2}>, \cdots,<u_{n}, \hat{a}_{n}>\right) \\
& =\bigotimes_{i=1}^{n}\left(\hat{a}_{(j)}\right)^{\frac{\varphi_{j}(\mu, N) \varphi_{(j)}(\nu, A)}{\sum_{j=1}^{n} \varphi_{j}(\mu, N) \varphi_{(j)}(\nu, A)}}
\end{aligned}
$$

Remark 10. If $\gamma=2$, then IGINSHGM operator degenerates to the Induced Interval Neutrosophic Shapley Hybrid Quadratic Geometric (IINSHQG) operator:

$$
\begin{aligned}
\operatorname{IINSHQG}( & \left.<u_{1}, \hat{a}_{1}\right\rangle,\left\langle u_{2}, \hat{a}_{2}>, \cdots,<u_{n}, \hat{a}_{n}>\right) \\
& =\frac{1}{2}\left({\underset{i=1}{\otimes}\left(2 \cdot \hat{a}_{(j)}\right)}^{\frac{\varphi_{j}(\mu, N) \varphi_{(j)}(\nu, A)}{\sum_{=1}^{n} \varphi_{j}(\mu, N) \varphi_{(j)}(\nu, A)}}\right) .
\end{aligned}
$$

From the above analysis, we know that IGINSHGM operator is the generalized form of IGINHGM, IINSHGM, and IINSHQG operators.

Similar to IGINSHAA operator, we can prove that IGINSHGM operator has idempotency, commutativity, monotonicity, and boundedness. It is omitted here.

\section{An approach to MADM under interval neutrosophic environment}

For a MADM problem with interval neutrosophic information, in which the criteria are interactive, suppose that $A=\left\{A_{1}, A_{2}, \cdots, A_{m}\right\}$ is a set of $m$ candidate alternatives, $C=\left\{C_{1}, C_{2}, \cdots, C_{n}\right\}$ is a set of $n$ attributes. Assume that $R=\left[r_{i j}\right]_{m \times n}$ is a decision matrix, where $\left.r_{i j}=<\left[T_{i j}^{L}, T_{i j}^{U}\right],\left[I_{i j}^{L}, I_{i j}^{U}\right],\left[F_{i j}^{L}, F_{i j}^{U}\right]\right)>$ $(i=1,2, \cdots, m ; j=1,2, \cdots, n)$ is the attribute value expressed by the interval neutrosophic information for alternative $A_{i}$ with respect to attribute $C_{j}$, satisfying $\left[T_{i j}^{L}, T_{i j}^{U}\right] \subseteq[0,1],\left[I_{i j}^{L}, I_{i j}^{U}\right] \subseteq[0,1],\left[F_{i j}^{L}, F_{i j}^{U}\right] \subseteq[0,1]$, and $0 \leq T_{i j}^{U}+I_{i j}^{U}+F_{i j}^{U} \leq 3$.

\subsection{The models based on cross entropy for obtaining the optimal fuzzy measures}

Due to time pressure, lack of knowledge, the expert's limited expertise about the complex problems, the attribute weights are usually incompletely known. Because the cross entropy is a very important method to obtain the weight vector [44], the models based on the cross entropy are respectively established to obtain the optimal fuzzy measures on the attribute and ordered sets.

Here, the Shapley values can overall reflect the correlations between criteria, and it is regarded as the criteria weights.

Firstly, we use the approach proposed by Zhang et al. [44] to obtain optimal fuzzy measure $\nu$ on attribute set $C$. Suppose that $R^{+}=\left(r_{1}^{+}, r_{2}^{+}, \cdots, r_{n}^{+}\right)$and $R^{-}=$ $\left(r_{1}^{-}, r_{2}^{-}, \cdots, r_{n}^{-}\right)$represent the positive and negative ideal alternatives, respectively, where:

$$
\begin{aligned}
& r_{j}^{+}=\{ {\left[\max _{1 \leq i \leq m} T_{i j}^{L}, \max _{1 \leq i \leq m} T_{i j}^{U}\right], } \\
& {\left[\min _{1 \leq i \leq m} I_{i j}^{L}, \min _{1 \leq i \leq m} I_{i j}^{U}\right], } \\
& {\left.\left[\min _{1 \leq i \leq m} F_{i j}^{U}, \min _{1 \leq i \leq m} F_{i j}^{U}\right]\right\}, } \\
& r_{j}^{-}=\left\{\left[\min _{1 \leq i \leq m} T_{i j}^{L}, \min _{1 \leq i \leq m} T_{i j}^{U}\right],\right. \\
& {\left[\max _{1 \leq i \leq m} I_{i j}^{L}, \max _{1 \leq i \leq m} I_{i j}^{U}\right], } \\
& {\left.\left[\max _{1 \leq i \leq m} F_{i j}^{U}, \max _{1 \leq i \leq m} F_{i j}^{U}\right]\right\} . }
\end{aligned}
$$

We can get the performance of alternative $A_{i}$ with respect to attribute $C_{j}$ as follows:

$$
D_{i j}=\frac{D_{i j}^{-}}{D_{i j}^{-}+D_{i j}^{+}},
$$

where $D_{i j}^{-}$and $D_{i j}^{+}$are the degrees of discriminations of $A_{i}$ from positive ideal solution $r_{j}^{+}$and form negative ideal solution $r_{j}^{-}$with respect to attribute $C_{j}$, respectively.

If the criteria weights are incompletely known, according to the model established by Zhang et al. [44], we can construct the following model to obtain optimal fuzzy measure $\nu$ on attribute $C$ :

$$
\begin{array}{ll}
\max & \sum_{i=1}^{m} \sum_{j=1}^{n} \frac{D_{i j}^{-}}{D_{i j}^{-}+D_{i j}^{+}} \varphi_{c_{j}}(\nu, C), \\
\text { s.t. } & \begin{cases}\nu(\Phi)=0, & \nu(C)=1 \\
\nu(G) \leq \nu(F) \forall G, & F \subseteq C, \quad G \subseteq F \\
\nu\left(C_{j}\right) \in W c_{j}, & j=1,2, \cdots, n\end{cases}
\end{array}
$$

where $\varphi_{c_{j}}(\nu, C)$ is the Shapley value of attribute $C_{j}$ with respect to fuzzy measure $\nu, W c_{j}(j=1,2, \cdots, n)$ is the known weight information. If the criteria weights are completely unknown, $\nu\left(c_{j}\right) \in W c_{j}(j=1,2, \cdots, n)$ in the above model should be omitted.

Then, we consider how to get optimal fuzzy measure $\mu$ on ordered set $N=\{1,2, \cdots, n\}$. If the weight information about ordered positions is partly known, the following model can be constructed to obtain optimal fuzzy measure $\mu$ on ordered set $N$ :

$$
\max \sum_{i=1}^{m} \sum_{j=1}^{n} \frac{D_{i(j)}^{-}}{D_{i(j)}^{-}+D_{i(j)}^{+}} \varphi_{j}(\mu, N),
$$




$$
\text { s.t. } \begin{cases}\mu(\Phi)=0, & \mu(N)=1 \\ \mu(G) \leq \mu(F) \forall G, & F \subseteq N, \quad G \subseteq F \\ \mu(j) \in W_{j}, & j=1,2, \cdots, n\end{cases}
$$

where $\varphi_{j}(\mu, N)$ is the Shapley value of the $j$ th position with respect to fuzzy measure $\mu$, and $(\cdot)$ is a permutation on $N$ for each $i=1,2, \cdots, m$, such that $\frac{D_{i(j)}^{-}}{D_{i(j)}^{-}+D_{i(j)}^{+}}$ is the $j$ th largest value of $\frac{D_{i j}^{-}}{D_{i j}^{-}+D_{i j}^{+}}$. It should be noted that if the weight information for the ordered positions is completely unknown, $\mu(j) \in W_{j}(j=1,2, \cdots, n)$ in the above model should be omitted.

\subsection{The decision procedure}

According to above models and induced generalized interval neutrosophic Shapley hybrid operators, we propose a procedure to handle MADM problems in which the attribute values are in the form of INNs, and the weight information for attribute and ordered positions is incompletely known. The steps are given as follows:

- Step 1: Evaluate the alternatives with respect to the criteria to construct the interval neutrosophic matrix $R=\left[r_{i j}\right]_{m \times n}$;

- Step 2: Utilize Eq. (31) to calculate performance of alternative $A_{i}(i=1,2, \cdots, m)$ with respect to attribute $C_{j}(j=1,2, \cdots, n)$;

- Step 3: Utilize model (32) to obtain optimal fuzzy measure $\nu$ on attribute $C$ and calculate their Shapley values;

- Step 4: Utilize model (33) to obtain the optimal fuzzy measure, $\mu$, on ordered set, $N$, and calculate their Shapley values;

- Step 5: Utilize IGINSHAA operator or IGINSHGM operator to determine comprehensive interval neutrosophic value, $\tilde{r}_{i}$, of each alternative, $A_{i}(i=$ $1,2, \cdots, m)$;

- Step 6: Utilize Definition 5 to construct the possibility matrix of the score function value;
- Step 7: Rank all the alternatives $\left\{a_{1}, a_{2}, \cdots, a_{n}\right\}$ by comparison rules of interval neutrosophic numbers and select the best alternative(s);

- Step 8: End.

\section{An illustrative example}

Let us consider the MADM problems with respect to a manufacturing company to evaluate the global suppliers based on the core competencies of suppliers (adapted from [45]). Suppose that there are four suppliers whose core competencies are evaluated by the following four attributes $C=\left\{C_{1}, C_{2}, C_{3}, C_{4}\right\}$ : the level of technology innovation $\left(C_{1}\right)$; the control ability of flow $\left(C_{2}\right)$; the ability of management $\left(C_{3}\right)$; and the level of service $\left(C_{4}\right)$. The evaluated value of supplier $A_{i}(i=1,2,3,4)$ with respect to $C_{j}(j=1,2,3,4)$ can be expressed by interval neutrosophic number of:

$$
\begin{aligned}
r_{i j}= & \left.<\left[T_{i j}^{L}, T_{i j}^{U}\right],\left[I_{i j}^{L}, I_{i j}^{U}\right],\left[F_{i j}^{L}, F_{i j}^{U}\right]\right) \\
& >(i=1,2, \cdots, m ; j=1,2, \cdots, n) .
\end{aligned}
$$

Interval neutrosophic matrix $R=\left[r_{i j}\right]_{m \times n}$ is listed in Table 1. Assume that the importance of attributes is respectively given as $\omega_{1}=[0.3,0.4], \omega_{2}=[0.15,0.25]$, $\omega_{1}=[0.2,025]$, and $\omega_{4}=[0.25,0.3]$. Furthermore, the importance of the ordered positions is, respectively, defined by $w_{1}=[0.1,0.2], w_{2}=[0.2,0.3], w_{3}=$ $[0.3,0.4]$, and $w_{4}=[0.2,0.3]$.

\subsection{The evaluation steps by IGINSHAA operator}

- Step 1: Utilize Eq. (31) to calculate performance of alternative $A_{i}$ with respect to attribute $C_{j}$. We can get:

$$
\frac{D_{i j}^{-}}{D_{i j}^{-}+D_{i j}^{+}}=\left(\begin{array}{cccc}
0.0659 & 0.7709 & 0.7244 & 0.0567 \\
0.5922 & 0.5886 & 1.0000 & 0.0230 \\
0.8043 & 1.0000 & 0.0000 & 1.0000 \\
0.6951 & 0.0000 & 0.6994 & 0.9759
\end{array}\right) .
$$

Table 1. Interval neutrosophic decision matrix.

\begin{tabular}{ccc}
\hline & $\boldsymbol{C}_{\mathbf{1}}$ & $\boldsymbol{C}_{\mathbf{2}}$ \\
\hline$A_{1}$ & $\{[0.3,0.5],[0.2,0.3],[0.2,0.5]\}$ & $\{[0.3,0.5],[0.1,0.3],[0.3,0.5]\}$ \\
$A_{2}$ & $\{[0.4,0.5],[0.1,0.2],[0.3,0.4]\}$ & $\{[0.2,0.4],[0.1,0.2],[0.3,0.5]\}$ \\
$A_{3}$ & $\{[0.3,0.5],[0.1,0.2],[0.2,0.3]\}$ & $\{[0.3,0.5],[0.1,0.2],[0.3,0.5]\}$ \\
$A_{4}$ & $\{[0.4,0.6],[0.2,0.3],[0.2,0.3]\}$ & $\{[0.2,0.4],[0.2,0.3],[0.3,0.5]\}$ \\
\hline & $\boldsymbol{C}_{\mathbf{3}}$ & $\boldsymbol{C}_{\mathbf{4}}$ \\
\hline$A_{1}$ & $\{[0.5,0.6],[0.1,0.2],[0.1,0.3]\}$ & $\{[0.3,0.5],[0.2,0.4],[0.2,0.3]\}$ \\
$A_{2}$ & $\{[0.6,0.7],[0.1,0.2],[0.1,0.2]\}$ & $\{[0.4,0.5],[0.3,0.4],[0.2,0.4]\}$ \\
$A_{3}$ & $\{[0.4,0.6],[0.2,0.4],[0.2,0.3]\}$ & $\{[0.6,0.7],[0.1,0.2],[0.1,0.3]\}$ \\
$A_{4}$ & $\{[0.5,0.7],[0.1,0.2],[0.2,0.3]\}$ & $\{[0.5,0.7],[0.1,0.2],[0.1,0.3]\}$ \\
\hline & &
\end{tabular}


- Step 2: Utilize model (32) to obtain the optimal fuzzy measures on attribute set $C$. The programming model is shown as follows:

$$
\begin{aligned}
& \max =-0.03055\left(\nu\left(c_{1}\right)-\nu\left(c_{2}, c_{3}, c_{4}\right)\right) \\
& +0.03679\left(\nu\left(c_{2}\right)-\nu\left(c_{1}, c_{3}, c_{4}\right)\right) \\
& +0.05824\left(\nu\left(c_{3}\right)-\nu\left(c_{1}, c_{2}, c_{4}\right)\right) \\
& \quad-0.06448\left(\nu\left(c_{4}\right)-\nu\left(c_{1}, c_{2}, c_{3}\right)\right) \\
& \quad+0.00312\left(\nu\left(c_{1}, c_{2}\right)-\nu\left(c_{3}, c_{4}\right)\right) \\
& \quad+0.01385\left(\nu\left(c_{1}, c_{3}\right)-\nu\left(c_{2}, c_{4}\right)\right) \\
& \quad-0.04752\left(\nu\left(c_{1}, c_{4}\right)-\nu\left(c_{2}, c_{3}\right)\right)+2.24908, \\
& \begin{cases}\nu\left(c_{1},\right. & \left.c_{2}, c_{3}, c_{4}\right)=1 \quad u(\Phi)=0 \\
\nu(G) \leq \nu(F) \forall F, & G \subseteq\left\{c_{1}, c_{2}, c_{3}, c_{4}\right\} \\
\nu\left(c_{1}\right) & \in[0.3,0.4], \quad \nu\left(c_{2}\right) \in[0.15,0.25] \\
\nu\left(c_{3}\right) & \in[0.2,0.25], \quad \nu\left(c_{4}\right) \in[0.25,0.3]\end{cases}
\end{aligned}
$$

Solving the above model by Lingo software, we can obtain:

$$
\begin{gathered}
\begin{array}{c}
\nu\left(c_{1}\right)=\nu\left(c_{1}, c_{2}\right)=\nu\left(c_{1}, c_{3}\right)=\nu\left(c_{1}, c_{4}\right) \\
=\nu\left(c_{1}, c_{2}, c_{4}\right)=\nu\left(c_{1}, c_{3}, c_{4}\right)=0.3 \\
\nu\left(c_{2}\right)=\nu\left(c_{3}\right)=\nu\left(c_{4}\right)=\nu\left(c_{2}, c_{4}\right)=\nu\left(c_{3}, c_{4}\right)=0.2 \\
\nu\left(c_{2}, c_{3}\right)=\nu\left(c_{1}, c_{2}, c_{3}\right)=\nu\left(c_{2}, c_{3}, c_{4}\right) \\
=\nu\left(c_{1}, c_{2}, c_{3}, c_{4}\right)=1 .
\end{array}
\end{gathered}
$$

By Eq. (21), we can obtain the following attribute Shapley values:

$$
\begin{aligned}
& \varphi_{c_{1}}(\nu, C)=0.0958, \quad \varphi_{c_{2}}(\nu, C)=0.4208, \\
& \varphi_{c_{3}}(\nu, C)=0.4208, \quad \varphi_{c_{4}}(\nu, C)=0.0625 .
\end{aligned}
$$

- Step 3: Utilize model (33) to obtain optimal fuzzy measure, $\mu$, on the ordered set $N=\{1,2, \cdots, n\}$. The programming model is shown as follows:

$$
\begin{aligned}
\max = & 0.49925(\mu(1)-\mu(2,3,4)) \\
& +0.25563(\mu(c 2)-\mu(1,3,4)) \\
& -0.03175(\mu(3)-\mu(1,2,4)) \\
& -0.72313(\mu(4)-\mu(1,2,3)) \\
& +0.37744(\mu(1,2)-\mu(3,4))
\end{aligned}
$$

$$
\begin{aligned}
& +0.23375(\mu(1,3)-\mu(2,4)) \\
& -0.11194(\mu(1,4)-\mu(2,3))+2.24908
\end{aligned}
$$

$$
\begin{cases}\mu(1,2,3,4)=1 & \mu(\Phi)=0 \\ \mu(G) \leq \nu(G) \forall G, & F \subseteq\{1,2,3,4\} \\ & G \subseteq F \\ \mu(1) \in[0.1,0.2], & \mu(2) \in[0.2,0.3], \\ \mu(3) \in[0.3,0.4], & \mu(4) \in[0.2,0.3]\end{cases}
$$

Solving the above model by Lingo software, we can obtain:

$$
\begin{aligned}
& \mu(1)=\mu(4)=\mu(1,4)=0.2, \\
& \mu(2)=\mu(3)=\mu(1,3)=\mu(2,3)=\nu(2,4)=\mu(3,4) \\
& =\mu(1,3,4)=\mu(2,3,4)=0.3, \\
& \mu(1,2)=\mu(1,2,4)=\mu(1,2,3)=\mu(1,2,3,4)=1 .
\end{aligned}
$$

By Eq. (21), we get the following position Shapley values:

$$
\begin{array}{ll}
\varphi_{1}(\mu, N)=0.4000, & \varphi_{2}(\mu, N)=0.4500, \\
\varphi_{3}(\mu, N)=0.1000, \quad \varphi_{4}(\mu, N)=0.0500 .
\end{array}
$$

- Step 4: Utilize IGINSHAA operator to get the comprehensive interval neutrosophic value $\tilde{r}_{i}$ of each alternative $A_{i}(i=1,2,3,4)$. Suppose that $\lambda=1$, we can obtain:

$$
\begin{aligned}
\tilde{r}_{1}=< & {[0.4106,0.5539],[0.1024,0.2444], } \\
& {[0.1687,0.3834]>, } \\
\tilde{r}_{2}=< & {[0.5179,0.6315],[0.1013,0.2017], } \\
& {[0.1452,0.2633]>, } \\
\tilde{r}_{3}=< & {[0.4583,0.6073],[0.1066,0.2133], } \\
& {[0.1766,0.3742]>, } \\
\tilde{r}_{4}=< & {[0.4757,0.6780],[0.1091,0.2104], } \\
& {[0.1929,0.3135]>. }
\end{aligned}
$$

- Step 5: Utilize Definition 5 to construct the possibility matrix of the score function value, then we can obtain:

$\left[\begin{array}{cccc}0.5 & 0.2763 & 0.4321 & 0.3581 \\ 0.7237 & 0.5 & 0.6546 & 0.5727 \\ 0.5679 & 0.3454 & 0.5 & 0.4243 \\ 0.6419 & 0.4273 & 0.5757 & 0.5\end{array}\right]$.


- Step 6: Get the order of the alternatives, to have:

$$
a_{2} \succ a_{4} \succ a_{3} \succ a_{1} .
$$

Thus, the best alternative is $a_{2}$.

\subsection{The evaluation steps by IGINSHGM operator}

- Step 1-Step 3: See the above Steps 1 to 3;

- Step 4: Utilize IGINSHGM operator to get comprehensive interval neutrosophic value $\tilde{r}_{i}$ of each alternative $A_{i}(i=1,2,3,4)$ (suppose that $\left.\lambda=1\right)$, then we can get:

$$
\begin{aligned}
\tilde{r}_{1}=< & {[0.3895,0.5488],[0.1036,0.2515], } \\
& {[0.2004,0.4045]>, } \\
\tilde{r}_{2}=< & {[0.4658,0.6011],[0.1027,0.2028], } \\
& {[0.1732,0.2968]>, } \\
\tilde{r}_{3}=< & {[0.4158,0.5882],[0.1098,0.2210], } \\
& {[0.2054,0.3948]>, } \\
\tilde{r}_{4}=< & {[0.4581,0.6631],[0.1132,0.2132], } \\
& {[0.1996,0.3199]>. }
\end{aligned}
$$

- Step 5: Utilize Definition 5 to construct the possibility matrix of the score function value, then we can obtain:

$$
\left[\begin{array}{cccc}
0.5 & 0.3202 & 0.4520 & 0.3415 \\
0.6789 & 0.5 & 0.6313 & 0.5102 \\
0.5480 & 0.3687 & 0.5 & 0.3873 \\
0.6585 & 0.4898 & 0.6127 & 0.5
\end{array}\right] .
$$

- Step 6: Get the order of the alternatives, then we have:

$$
a_{2} \succ a_{4} \succ a_{3} \succ a_{1} .
$$

Thus, the best alternative is $a_{2}$.

\subsection{Analysis of the influence of parameter $\lambda$}

In order to demonstrate the influence of parameter $\lambda$ on decision-making results, we use different values $\lambda$ in IGINSHAA and IGINSHGM operators to rank the alternatives. The ranking results are listed in Table 2.

\begin{tabular}{|c|c|c|}
\hline$\lambda$ & $\begin{array}{c}\text { Ranking by } \\
\text { IGINSHAA } \\
\text { operator }\end{array}$ & $\begin{array}{c}\text { Ranking by } \\
\text { IGINSHGM } \\
\text { operator }\end{array}$ \\
\hline $1 / 2$ & $a_{2} \succ a_{4} \succ a_{3} \succ a_{1}$ & $a_{4} \succ a_{2} \succ a_{3} \succ a_{1}$ \\
\hline 1 & $a_{2} \succ a_{4} \succ a_{3} \succ a_{1}$ & $a_{2} \succ a_{4} \succ a_{3} \succ a_{1}$ \\
\hline 2 & $a_{2} \succ a_{4} \succ a_{3} \succ a_{1}$ & $a_{2} \succ a_{4} \succ a_{3} \succ a_{1}$ \\
\hline 5 & $a_{2} \succ a_{4} \succ a_{3} \succ a_{1}$ & $a_{4} \succ a_{2} \succ a_{1} \succ a_{3}$ \\
\hline 10 & $a_{2} \succ a_{3} \succ a_{4} \succ a_{1}$ & $a_{4} \succ a_{2} \succ a_{1} \succ a_{3}$ \\
\hline
\end{tabular}

As we can see from Table 2, the ranking results of the alternatives are different for the different values $\lambda$ in IGINSHAA and IGINSHGM operators. But, the best alternative from the different values $\lambda$ is the same in IGINSHAA operator. Thus, the individual or organization can properly select the favorable alternative in terms of his interest and actual needs. In general, we can get $\lambda=1$.
Table 2. Ordering of the alternatives by utilizing the different $\lambda$ in IGINSHAA and IGINSHGM operators.

\subsection{Comparison of the existing methods}

In order to demonstrate the practicality and effectiveness of the developed method in this paper, we can compare the proposed method with the existing method proposed by Zhang and $\mathrm{Wu}$ [46]. The steps are shown as follows:

- Step 1: Utilize the maximization deviation method to determine the attribute weights. The model is established as follows:

$$
\begin{aligned}
& \max =1.0699 w\left(c_{1}\right)+0.7587 w\left(c_{2}\right)+1.1132 w\left(c_{3}\right) \\
& +1.5471 w\left(c_{4}\right), \\
& \left\{\begin{array}{l}
w\left(c_{1}\right)+w\left(c_{2}\right)+w\left(c_{3}\right)+w\left(c_{4}\right)=1 \\
w\left(c_{1}\right) \in[0.3,0.4], \quad w\left(c_{2}\right) \in[0.15,0.25] \\
w\left(c_{3}\right) \in[0.2,0.25], \quad w\left(c_{4}\right) \in[0.25,0.3]
\end{array}\right.
\end{aligned}
$$

Solving this above model by Lingo software, we obtain:

$$
\begin{array}{ll}
w\left(c_{1}\right)=0.3, & w\left(c_{2}\right)=0.15, \\
w\left(c_{3}\right)=0.25, & w\left(c_{4}\right)=0.3 .
\end{array}
$$

- Step 2: Identify the positive ideal solution and negative ideal solution, then we can obtain:

$$
\begin{aligned}
\hat{r}^{+}= & (\{[0.4,0.6],[0.1,0.2],[0.2,0.3]\}, \\
& \{[0.3,0.5],[0.1,0.2],[0.3,0.5]\}, \\
& \{[0.6,0.7],[0.1,0.2],[0.1,0.2]\}, \\
& \{[0.6,0.7],[0.1,0.2],[0.1,0.3]\}), \\
\hat{r}^{-}= & (\{[0.3,0.5],[0.2,0.3],[0.3,0.5]\}, \\
& \{[0.2,0.4],[0.2,0.3],[0.3,0.5]\}, \\
& \{[0.4,0.6],[0.2,0.4],[0.2,0.3]\}, \\
& \{[0.3,0.5],[0.3,0.4],[0.2,0.4]\}) .
\end{aligned}
$$

- Step 3: Determine the distance between each 
alternative and the positive ideal and negative ideal solutions, respectively, then we can get:

$$
\begin{aligned}
& D^{+}=(0.1118,0.0818,0.0527,0.0595) \\
& D^{-}=(0.0672,0.0808,0.1034,0.1114)
\end{aligned}
$$

- Step 4: Determine the closeness coefficient of each alternative to the ideal solution, then we get:

$$
\begin{array}{ll}
C C_{1}=0.3753, & C C_{2}=0.4967, \\
C C_{3}=0.6625, & C C_{4}=0.6518 .
\end{array}
$$

- Step 5: According to relative closeness coefficient, $C C_{i}(i=1,2,3,4)$, get the order of the alternatives:

$$
a_{3} \succ a_{4} \succ a_{2} \succ a_{1}
$$

So, the best alternative is $a_{3}$.

From the above analysis, we can see that the ranking results by the proposed methods in this paper are different from those obtained by the existing method. The reason may be that the existing method could not weight the input arguments' positions and capture their interrelationship, which may result in the unreasonable ranking results. However, the method proposed in this paper can effectively handle the interval neutrosophic MADM problems in which the attribute and ordered position weights are incompletely known, and attributes exist correlatively. So, we think the method developed in this paper is more suitable to handle this application example.

\section{Conclusion}

This paper developed an approach to interval neutrosophic MADM with interactive conditions and incomplete weight information. To get the comprehensive values, two types of interval neutrosophic hybrid aggregation operators were introduced. One is based on additive measures which could consider the importance of the aggregated interval neutrosophic arguments and their ordered positions; the other utilizes the Shapley function in regard to fuzzy measures which not only considers the importance of the aggregated interval neutrosophic arguments and their ordered positions, but also captures their interrelationship. To get the weight information, the programming models based on cross entropy were established.

It should be mentioned that the fuzzy measures can well process the situations where the input arguments are correlative, but they are determined by ascertaining $2^{n}-2$ parameters for $n$ criteria. To reduce the complexity of calculating the fuzzy measures on the given set, it shall be significant to research into some special kinds of fuzzy measures, such as $\lambda$ fuzzy measures, $k$-additive measure, and $p$-symmetry measures. In addition, in future research, we shall concentrate on extending the ideas of this paper by using the refined neutrosophic set as a generalized form of neutrosophic set [47].

\section{Acknowledgment}

This paper is supported by the National Natural Science Foundation of China (Nos. 71471172 and 71271124), the Special Funds of Taishan Scholars Project of Shandong Province, National Soft Science Project of China (2014GXQ4D192), the Humanities and Social Sciences Research Project of Ministry of Education of China (No. 13YJC630104), Shandong Provincial Social Science Planning Project (No. 15BGLJ06). The authors also would like to express thanks to the anonymous reviewers and editors for their very helpful comments that improved the paper.

\section{References}

1. Zadeh, L.A. "Fuzzy sets", Information and Control, 8(3), pp. 338-353 (1965).

2. Gau, W.L. and Buehrer, D.J. "Vague sets", IEEE Transactions on Systems, Man, and Cybernetics, Part B: Cybernetics, 23, pp. 610-614 (1993).

3. Atanassov, K.T. "Intuitionistic fuzzy sets", Fuzzy Sets and Systems, 20(1), pp. 87-96 (1986).

4. Smarandache, F., Neutrosophy. Neutrosophic Probability, Set, and Logic, American Research Press, Rehoboth (1998).

5. Ye, S., Fu, J. and Ye, J. "Medical diagnosis using distance-based similarity measures of single valued neutrosophic multisets", Neutrosophic Sets and Systems, 7, pp. 47-52 (2015).

6. Ye, S. and Ye, J. "Dice similarity measure between single valued neutrosophic multisets and its application in medical diagnosis", Neutrosophic Sets and Systems, 6, pp. 49-54 (2014).

7. Salama, A.A., Smarandache, F. and Eisa, M. "Introduction to image processing via neutrosophic techniques", Neutrosophic Sets and Systems, 5, pp. 59-64 (2014).

8. Mukherjee, A. and Sarkar, S. "A new method of measuring similarity between two neutrosophic soft sets and its application in pattern recognition problems", Neutrosophic Sets and Systems, 8, pp. 63-68 (2015).

9. Deli, I., Broumi, S. and Ali, M. "Neutrosophic soft multi-set theory and its decision making", Neutrosophic Sets and Systems, 5, pp. 65-76 (2014).

10. Mondal, K. and Pramanik, S. "Neutrosophic decision making model of school choice", Neutrosophic Sets and Systems, 7, pp. 62-68 (2015). 
11. Mondal, K. and Pramanik, S. "Rough neutrosophic multi-attribute decision-making based on rough accuracy score function", Neutrosophic Sets and Systems, 8, pp. 14-21 (2015).

12. Bausys, R., Zavadskas, E.K. and Kaklauskas, A. "Application of neutrosophic set to multicriteria decision making by COPRAS", Economic Computation and Economic Cybernetics Studies and Research, 49(2), pp. 91-105 (2015).

13. Chakraborty, S., Zavadskas, E.K. and Antucheviciene, J. "Applications of WASPAS method as a multicriteria decision-making tool", Economic Computation and Economic Cybemetics Studies and Research, 49(1), pp. 5-22 (2015).

14. Peng, J.J., Wang, J.E., Zhang, H.Y. and Chen, X.H. "An outranking approach for multi-criteria decisionmaking problems with simplified neutrosophic sets", Applied Soft Computing, 25, pp. 336-346 (2014).

15. Sun, H.X., Yang, H.X., Wu, J.Z. and Ouyang, Y. "Interval neutrosophic numbers Choquet integral operator for multi-criteria decision making", Journal of Intelligent \& Fuzzy Systems, 28(6), pp. 2443-2455 (2015).

16. Ye, J. "An extended TOPSIS method for multiple attribute group decision making based on single valued neutrosophic linguistic numbers", Journal of Intelligent \& Fuzzy Systems, 23(1), pp. 247-255 (2015).

17. Zavadskas, E.K., Bauiys, R.B., Lazauskas, M. and Rosen, M.A. "Sustainable assessment of alternative sites for the construction of a waste incineration plant by applying WASPAS method with single-valued neutrosophic set", Sustainability, 7(12), pp. 15923-15936 (2015).

18. Wang, H., Smarandache, F., Zhang, Y.Q. and Sunderraman, R. "Single valued neutrosophic sets", Multispace and Multistructure, 4, pp. 410-413 (2010).

19. Majumdar, P. and Samanta, S.K. "On similarity and entropy of neutrosophic sets", Journal of Intelligent and Fuzzy Systems, 26(3), pp. 1245-1254 (2014).

20. Ye, J. "Single valued neutrosophic cross-entropy for multicriteria decision making problems", Applied Mathematical Modelling, 38, pp. 1170-1175 (2014).

21. Pătraşcu, V. "The neutrosophic entropy and its five components", Neutrosophic Sets and Systems, 7, pp. 40-46 (2015).

22. Biswas, P., Pramanik, S. and Bibhas, C.G. "Entropy based grey relational analysis method for multiattribute decision making under single valued neutrosophic assessments", Neutrosophic Sets and Systems, 2, pp. 102-110 (2014).

23. Biswas, P., Pramanik, S. and Bibhas, C.G. "TOPSIS method for multi-attribute group decision-making under single-valued neutrosophic environment", $\mathrm{Neu}$ ral Computing and Applications, 27(3), pp. 727-737 (2016).
24. Wang, H., Smarandache, F., Zhang, Y.Q. and Sunderraman, R., Interval Neutrosophic Sets and Logic: Theory and Applications in Computing, Hexis, Phoenix, Ariz, USA (2005).

25. Chi, P.P. and Liu, P.D. "An extended TOPSIS method for the multiple attribute decision making problems based on interval neutrosophic set", Neutrosophic Sets and Systems, 1, pp. 63-70 (2013) .

26. Liu, P.D. and Jin, F. "A multi-attribute group decision-making method based on weighted geometric aggregation operators of interval-valued trapezoidal fuzzy numbers", Applied Mathematical Modelling, 36, pp. 2498-2509 (2012).

27. Liu, P.D., Zhang, X. and Jin, F. "A multi-attribute group decision-making method based on intervalvalued trapezoidal fuzzy numbers hybrid harmonic averaging operators", Journal of Intelligent \& Fuzzy Systems, 23, pp. 159-168 (2012).

28. Liu, P.D., He, L. and Yu, X.C. "Generalized hybrid aggregation operators based on the 2-dimension uncertain linguistic information for multiple attribute group decision making", Group Decision and Negotiation, 25, pp. 103-126 (2016).

29. Liu, P.D. and Yu, X.C. "2-dimension uncertain linguistic power generalized weighted aggregation operator and its application for multiple attribute group decision making", Knowledge-based Systems, 57, pp. 69-80 (2014).

30. Liu, P.D. and Liu, Y. "An approach to multiple attribute group decision making based on intuitionistic trapezoidal fuzzy power generalized aggregation operator", International Journal of Computational Intelligence Systems, 7, pp. 291-304 (2014).

31. Ye, J. "A multicriteria decision-making method using aggregation operators for simplified neutrosophic sets", Journal of Intelligent and Fuzzy Systems, 26, pp. 24592466 (2014).

32. Liu, P.D. and Wang, Y.M. "Multiple attribute decision making method based on single-valued neutrosophic normalized weighted Bonferroni mean", Neural Computing and Applications, 25(7-8), pp. 2001-2010 (2014).

33. Zhang, H.Y., Wang, J.Q. and Chen, X.H. "Interval neutrosophic sets and their application in multicriteria decision making problems", The Scientific World Journal, 2014, pp. 1-15 (2014).

34. Ye, J. "Multiple attribute decision-making method based on the possibility degree ranking method and ordered weighted aggregation operators of interval neutrosophic numbers", Journal of Intelligent and Fuzzy Systems, 28, pp. 1307-1317 (2015).

35. Broumi, S. and Smarandache, F. "New distance and similarity measures of interval neutrosophic sets", Information Fusion, on 2014 17th International Conference, pp. 1-7 (2014).

36. Shang, X.G. and Jiang, W.S. "A note on fuzzy information measures", Pattern Recognition Letters, 18(5), pp. 425-432 (1997). 
37. Lin, J. "Divergence measures based on Shannon entropy", IEEE Transactions on Information Theory, 37(1), pp. 145-151 (1991).

38. Merigo, J.M. and Casanovas, M. "The induced generalized hybrid averaging operator and its application in financial decision making", International Journal of Business, Economics, Finance and Management Sciences, 2, pp. 95-101 (2009).

39. Lin, J. and Jiang, Y. "Some hybrid weighted averaging operators and their application to decision making", Information Fusion, 16, pp. 18-28 (2014).

40. Dyckhoff, H. and Pedrycz, W. "Generalized means as model of compensative connectives", Fuzzy Sets and Systems, 14(2), pp. 143-154 (1984).

41. Meng, F.Y., Tan, C.Q. and Zhang, Q. "The induced generalized interval-valued intuitionistic fuzzy hybrid Shapley averaging operator and its application in decision making", Knowledge-Based Systems, 42, pp. 9-19 (2013).

42. Sugeno, M. "Theory of fuzzy integral and its application", Doctorial Dissertation, Tokyo Institute of Technology (1974).

43. Shapley, L.S., A Value for n-Person Game, Princeton University Press, Princeton (1953).

44. Zhang, H.M. and Yu, L.Y. "MADM method based on cross entropy and extended TOPSIS with intervalvalued intuitionistic fuzzy sets", Knowledge-Based Systems, 30, pp. 115-120 (2012).

45. Tan, C.Q., Wu, D.S.D. and Ma, B.J. "Group decision making with linguistic preference relations with application to supplier selection", Expert Systems with Applications, 38(12), pp. 14382-14389 (2011).

46. Zhang, Z.M. and Wu, C. "A novel method for singlevalued neutrosophic multi-criteria decision making with incomplete weight information", Neutrosophic Sets and Systems, 4, pp. 35-49 (2014).

47. Smarandache, F. " $n$-valued refined neutrosophic logic and its applications in physics", Progress in Physics, 4, pp. 143-146 (2013).

\section{Biographies}

Peide Liu is Professor at the School of Management Science and Engineering in Shandong University of Finance and Economics, and Assistant Director of the Enterprise's Electronic-commerce Engineering Research Center of Shandong.

He has authored or co-authored more than 110 publications. His research interests include aggregation operators, fuzzy logic, fuzzy decision making, and their applications.

Guolin Tang is a master student at the School of Management Science and Engineering in Shandong University of Finance and Economics. He has authored or co-authored more than 6 publications. His research interests include aggregation operators, fuzzy logic, fuzzy decision making, and their applications.

Weilong Liu is Professor at the School of Management Science and Engineering in Shandong University of Finance and Economics, and Assistant director of the Enterprise's Electronic-commerce Engineering Research Center of Shandong.

He has authored or co-authored more than 20 publications. His research interests include information management, big data, fuzzy decision making, and their applications. 